\title{
14. SEDIMENT ACCUMULATION RATES OF THE LESSER ANTILLES INTRAOCEANIC ISLAND ARC, DEEP SEA DRILLING PROJECT LEG 78A ${ }^{1}$
}

\author{
Audrey Wright, Earth Sciences and Marine Studies, University of California at Santa Cruz ${ }^{2}$
}

\begin{abstract}
Bulk sediment accumulation rates and carbonate and carbonate-free accumulation rates corrected for tectonic tilting have been calculated for Leg 78A sediments. These rates are uniformly low, ranging from 0.1 to $6.8 \mathrm{~g} /\left(\mathrm{cm}^{2} \cdot 10^{3} \mathrm{yr}\right.$.), reflecting the pelagic-hemipelagic nature of all the sediments drilled in the northern Lesser Antilles forearc. Rates calculated for Sites 541 and $542\left[0.6-6.8 \mathrm{~g} /\left(\mathrm{cm}^{2} \cdot 10^{3} \mathrm{yr}\right.\right.$.)], located on the lower slope of the accretionary prism, are significantly greater than the Neogene rates calculated for oceanic reference Site $543\left[0.1-2.4 \mathrm{~g} /\left(\mathrm{cm}^{2} \cdot 10^{3}\right)\right]$. This difference could be the result of (1) tectonic thickening of accretionary prism sediments due to folding, small-scale faulting, and layer-parallel shortening; (2) deposition in shallower water farther above the CCD (carbonate compensation depth) resulting in preservation of a greater percentage of calcareous microfossils; or (3) a greater percentage of foraminiferal sediment gravity flows. Terrigenous turbidites are not documented in the Leg 78A area because of (1) great distance from South American sources; (2) damming effects of east-west trending tectonic elements; and (3) location on the Tiburon Rise (Site 543). This lack of terrigenous material, characteristic of intraoceanic convergent margins, suggests that published sedimentation models for active continental convergent margins with abundant terrigenous influxes are not applicable to intraoceanic convergent margin settings.
\end{abstract}

\section{INTRODUCTION}

Careful calculation of the changes in rates of deposition of sedimentary components with time provides valuable geologic information, such as variation in volcanic influx, frequency and volume of downslope sediment movement, and importance of tectonic thickening processes, when we attempt to reconstruct ancient depositional environments. Though sedimentation rates (in meters/Ma) are almost routinely calculated for DSDP cores (Davies et al., 1977), these rates are rarely corrected for downhole compaction-related changes in porosity or wet-bulk density. Making these corrections results in the calculation of sediment accumulation rates in $\mathrm{g} /\left(\mathrm{cm}^{2} \cdot 10^{3} \mathrm{yr}\right.$.) (c.f., van Andel et al., 1975; Worsley and Davies, 1979a and 1979b). Further corrections must be made to account for tectonic tilting, documented by nonhorizontal bedding attitudes (Shephard and McMillen, 1982). Variations in downhole porosity, wet-bulk density, and bedding attitudes can have a significant effect on calculated accumulation rates, and must be accounted for before interpreting sedimentation rate fluctuations either downhole at a single DSDP site or between sites drilled in different depositional settings.

Sediment accumulation rates, corrected for downhole porosity, wet-bulk density, and bedding attitude variations, have been calculated for sites drilled in the Lesser Antilles intraoceanic forearc region on DSDP Leg 78A (Fig. 1) and are presented in this chapter. Applying these corrections permits better interpretation of sedimentation rate fluctuations with time at each site and better

\footnotetext{
${ }^{1}$ Biju-Duval, B., Moore, J. C., et al., Init. Repts. DSDP, 78A: Washington (U.S. Govt. Printing Office).

2 Present address: Ocean Drilling Program, Texas A\&M University, 500 University Drive, West, College Station, TX 77848.
}

comparison of the sedimentation rates between the drilling sites.

\section{TECHNIQUES}

Bulk sediment accumulation rates $\left[\right.$ in $\mathrm{g} /\left(\mathrm{cm}^{2} \cdot 10^{3} \mathrm{yr}\right.$. $\left.)\right]$ were calculated at one-million-year (1-Ma) intervals with the equation presented by van Andel et al. (1975):

$$
R=(D-0.01025 P)(0.1 S)
$$

where $R=$ bulk sediment accumulation rate $\left[\mathrm{g} /\left(\mathrm{cm}^{2} \cdot 10^{3} \mathrm{yr}.\right)\right], D=$ wet bulk density $\left(\mathrm{g} / \mathrm{cm}^{3}\right), P=$ sediment porosity $(\%)$, and $S=$ sedimentation rate $(\mathrm{m} / \mathrm{Ma})$. This equation is based on the assumption that all downhole porosity and density changes are a result of postburial compaction, rather than cementation.

Downhole sedimentation rates (in $\mathrm{m} / \mathrm{Ma}$ ) were determined from age-versus-depth relationships documented in each hole. Age information was based primarily on biostratigraphic zonations determined using nannofossils (Bergen, this volume; Verbeck, 1976; Gartner, 1977; Bukry and Okada, 1980) and using radiolarians (Renz, this volume; Riedel and Sanfilippo, 1978; Sanfilippo and Riedel, 1982). The use of nannofossil and radiolarian biostratigraphic zones permitted finer time resolution than would have been possible with available foraminiferal biostratigraphic zone information. The subseafloor depth of biostratigraphic zone boundaries was determined according to the rules outlined by Davies et al. (1977) and Whitman and Davies (1979).

Absolute age boundaries of biostratigraphic zones were interpolated to permit calculation of sedimentation rates (in $\mathrm{m} / \mathrm{Ma}$ ) at $1-\mathrm{Ma}$ intervals. These sedimentation rate determinations are poorest in cored sections with low recovery, in barren cores (especially in sections where the sedimentation rate is low), and in intervals where the relationship of absolute age to biostratigraphic zonation is uncertain. Displacements along small-scale faults documented by biostratigraphic zone repetitions in Sites 541 and 542 were accounted for when calculating sedimentation rates within those biostratigraphic intervals. No corrections could be made to account for displacements along faults too small to be documented by any biostratigraphic zone inversion.

The average wet-bulk density and average sediment porosity for each 1-Ma interval was estimated from smoothed curves of the Gamma Ray Attenuation Porosity Evaluator (GRAPE) measurements made on board ship (Marlow et al., this volume). Evans and Cotterell (1970), Bader (1970), and Boyce (1976) describe the techniques used in calculating GRAPE porosities and wet-bulk densities. Bennett and 

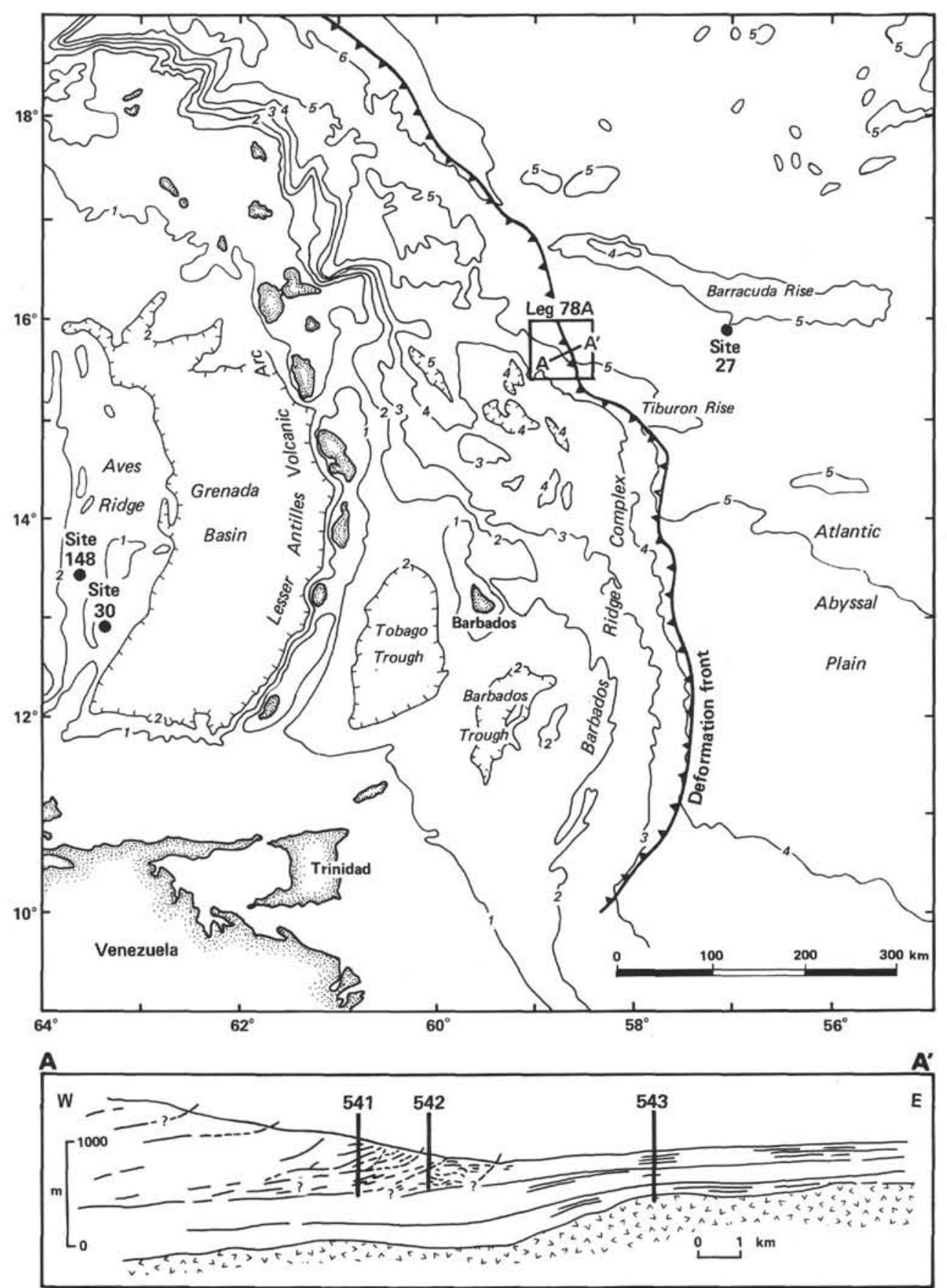

Figure 1. Location of Leg 78A area in the Lesser Antilles intraoceanic island arc. The schematic crosssection $\mathrm{A}-\mathrm{A}^{\prime}$ showing site locations extends from the Atlantic abyssal plain into the deformed accretionary prism and was compiled from seismic lines A1C and A1D (see Sites 541-543 reports, this volume) and drilling results. Sites 541 and 542 are located 3 and $1.5 \mathrm{~km}$, respectively, west of the deformation front on the toe of the accretionary prism and document the nature of the offscraped sedimentary section. Site 543 , located $3.5 \mathrm{~km}$ east of the deformation front, serves as the oceanic reference site.

Keller (1973) discuss the reliability of GRAPE determinations. Figure 2 (Site 541), Figure 3 (Site 542), and Figure 4 (Site 543) show the smoothed porosity and wet-bulk density curves used to estimate downhole porosity and density values.

After calculating bulk sediment accumulation rates by correcting for downhole compaction-related changes in porosity and density, these rates were further corrected for tectonic tilting, as indicated by average true bedding dips (Cowan et al., this volume). Bedding dip corrections are important at Sites 541 and 542 drilled within the accretionary prism where measured bedding attitudes range from 16 to $25^{\circ}$. The bedding attitudes observed at Site 543 were everywhere less than or equal to $5^{\circ}$, making tectonic tilting corrections unimportant at that site. Dip-corrected bulk sediment accumulation rates were made from bulk sediment accumulation rates in the following manner:

$$
D R=R(\cos B)
$$




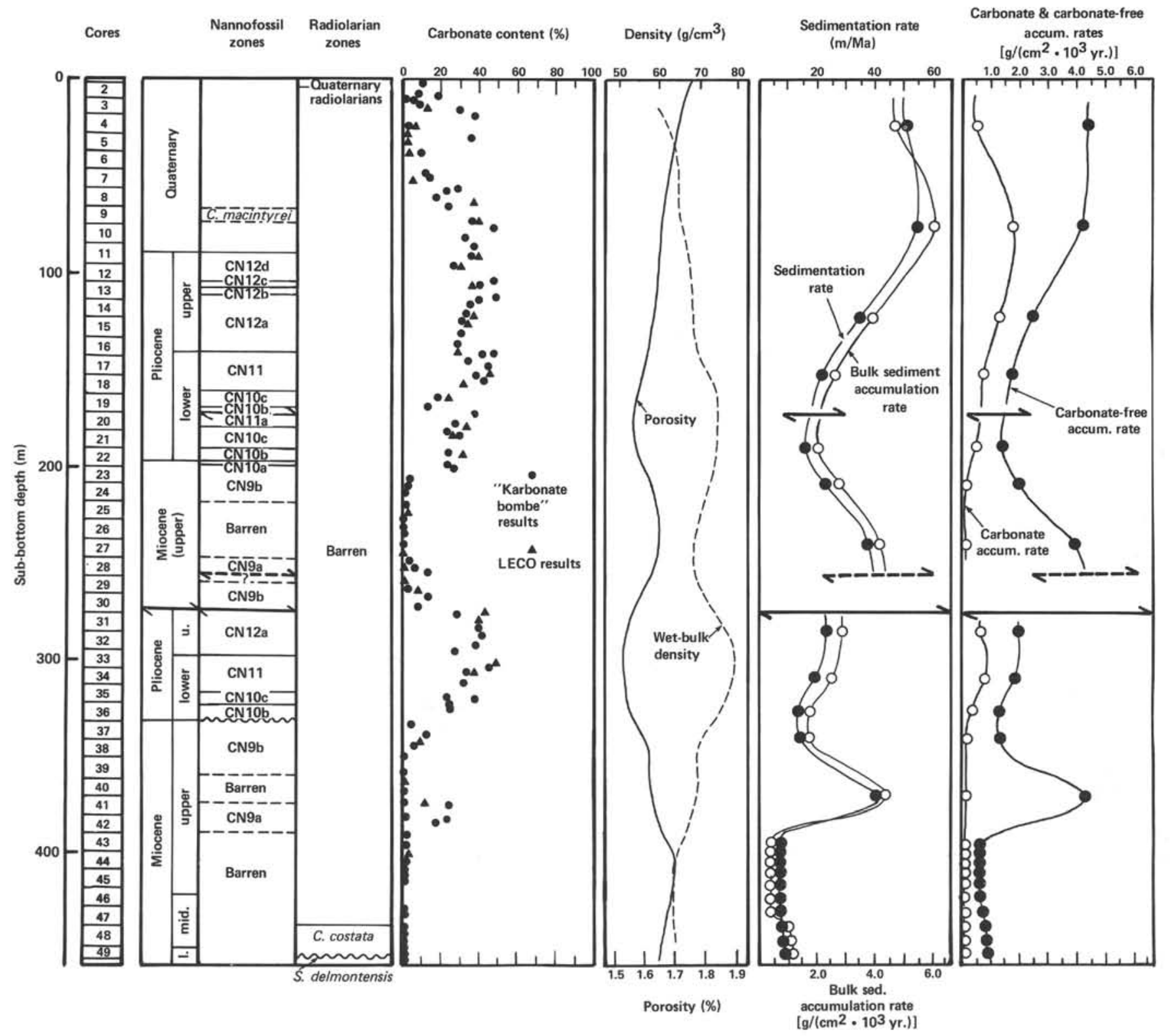

Figure 2. Site 541 nannofossil and radiolarian biostratigraphic zonations, calcium carbonate contents, porosity and wet-bulk density curves, ageversus-depth (sedimentation rate) curve, dip-corrected bulk sediment accumulation rate curve, and carbonate and carbonate-free accumulation rate curves. (Breaks in the sediment accumulation rate curves marked by heavy arrows indicate locations of faults documented by biostratigraphic inversions.)

where $D R=$ dip-corrected bulk sediment accumulation rate $\left[\mathrm{g} /\left(\mathrm{cm}^{2}\right.\right.$. $\left.\left.10^{3} \mathrm{yr}.\right)\right], R=$ bulk sediment accumulation rate $\left[\mathrm{g} /\left(\mathrm{cm}^{2} \cdot 10^{3} \mathrm{yr}.\right)\right]$ determined by the van Andel et al. (1975) equation cited earlier, and $B=$ average bedding dip $\left({ }^{\circ}\right)$.

Dip-corrected bulk sediment accumulation rates were divided into carbonate and carbonate-free accumulation rates based on the average $\mathrm{CaCO}_{3}$ percentage of each 1-Ma interval in the following manner:

$$
\begin{aligned}
C D R & =D R(0.01 C) \\
N C D R & =D R-C D R
\end{aligned}
$$

where $C D R=$ dip-corrected carbonate accumulation rate $\left[\mathrm{g} /\left(\mathrm{cm}^{2}\right.\right.$. $10^{3}$ yr.)], $N C D R=$ dip-corrected carbonate-free accumulation rate $\left[\mathrm{g} /\left(\mathrm{cm}^{2} \cdot 10^{3}\right.\right.$ yr. $\left.)\right], D R=$ dip-corrected bulk sediment accumulation rate $\left[\mathrm{g} /\left(\mathrm{cm}^{2} \cdot 10^{3} \mathrm{yr}.\right)\right]$ just discussed, and $C=$ average carbonate percentage (\%) of the 1-Ma interval. The downhole carbonate content of sediments at each site was determined in two different ways: (1) on board ship with the "Karbonate Bombe" (Müller and Gastner, 1974; Figs. 2, 3, and 4); and (2) in shore-based studies with the LECO Carbon Analyzer (Bader, 1970; Bode and Cronan, 1973; Boyce and Bode, 1973; Figs. 2, 3, and 4). The "Karbonate Bombe" permits rapid calculation of the carbonate content of sediment samples; the LECO Carbon Analyzer allows determination of both the carbonate and organic carbon percentages present in a sample. Tables 1 and 2 list the "Karbonate Bombe" and the LECO carbonate data.

Figure 2 (Site 541), Figure 3 (Site 542), and Figure 4 (Site 543) show dip-corrected bulk sediment accumulation rate curves, age-versus-depth (sedimentation rate) curves, carbonate and carbonate-free accumulation rate curves, calcium carbonate contents, porosity and wet-bulk density curves, and nannofossil and radiolarian biostratigraphic zonations. Table 3 lists sedimentation rates, porosity and wet-bulk density values, bulk sediment accumulation rates, approximate bedding attitudes, dip-corrected bulk sediment accumulation rates, calcium carbonate percentages, and carbonate and carbonate-free accumulation rates at one-million-year intervals for each site. 


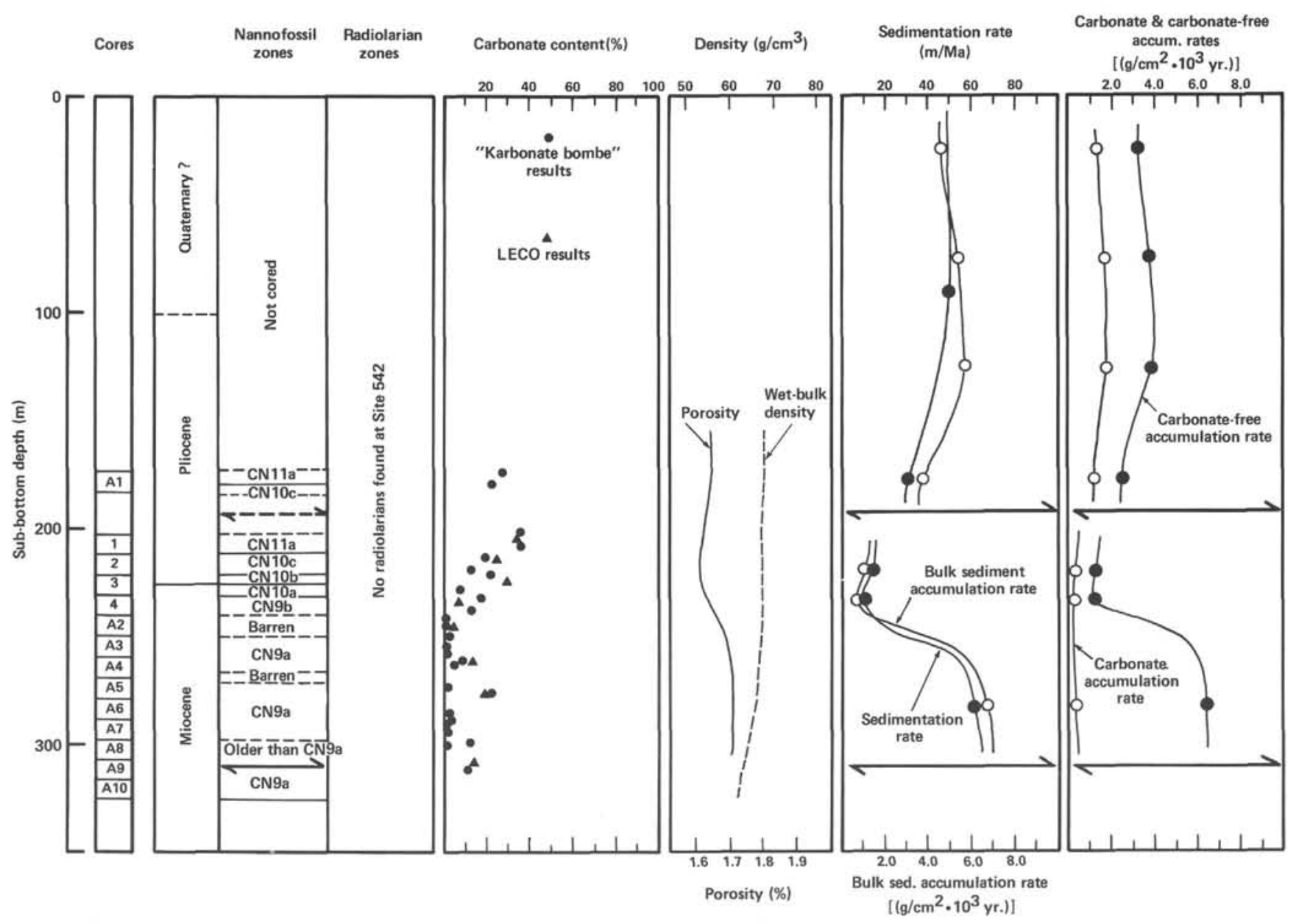

Figure 3. Site 542 nannofossil and radiolarian biostratigraphic zonations, calcium carbonate contents, porosity and wet-bulk density curves, ageversus-depth (sedimentation rate) curve, dip-corrected bulk sediment accumulation rate curve, and carbonate and carbonate-free accumulation rate curves. (Breaks in the sediment accumulation rate curves marked by heavy arrows indicate locations of faults documented by biostratigraphic inversions.)

\section{RESULTS}

\section{Site 541}

Site 541 was drilled in the Lesser Antilles lower slope environment, $3 \mathrm{~km}$ landward of the deformation front (Fig. 1). Sediments were cored continuously from the seafloor to a sub-bottom depth of $459 \mathrm{~m}$, bottoming in a lower Miocene radiolarian mudstone. Core recoveries ranged from 7 to $106 \%$, averaging $87 \%$. Site 541 sediments were divided into two tectonic units separated by a reverse fault at $276 \mathrm{~m}$ sub-bottom, which emplaced upper Miocene mud (Tectonic Unit A) over lower upper Pliocene nannofossil mudstone (Tectonic Unit B). Additional, smaller-scale biostratigraphic inversions are documented between Cores 541-19 and 541-20 and Cores 541-28 and 541-29 (Bergen, this volume). Sediment accumulation rates were determined separately for Tectonic Units A and B, and corrections were made for the smaller-scale documented biostratigraphic inversions (Table 3; Fig. 2). Intervals barren of all microfossils are found between 219 and $248 \mathrm{~m}$ (Cores 25-27), 362 and $376 \mathrm{~m}$ (Core 40-Sample 541-41-3, $150 \mathrm{~cm}$ ), and 388.5 and $438 \mathrm{~m}$ (Section 541-42-6 to Core 47) sub-bottom, and the 1-Ma time increments interpolated within these intervals are subsequently more approximate than in cored intervals containing abundant and well-preserved microfossils.

Tectonic Unit A sediments are Quaternary to upper Miocene. Quaternary (0-1.8 Ma) marly nannofossil oozes and nannofossil muds, drilled in the uppermost $91.5 \mathrm{~m}$ of the hole, have million-year time increment dip-corrected bulk sediment accumulation rates ranging from 4.9 to $6.1 \mathrm{~g} /\left(\mathrm{cm}^{2} \cdot 10^{3} \mathrm{yr}\right.$ ) (Table 3$)$. Pliocene $(1.8-5$ Ma) marly nannofossil oozes and nannofossil muds, drilled from 91.5 to $197 \mathrm{~m}$ sub-bottom, have smaller dip-corrected bulk sediment accumulation rates ranging from 1.9 to $3.8 \mathrm{~g} /\left(\mathrm{cm}^{2} \cdot 10^{3} \mathrm{yr}\right.$.) in million-year increments (Table 3). The Pliocene 4- to 5-Ma interval contains a repeated nannofossil zone documented by a biostratigraphic inversion between Cores 541-19 and 54120 . The sediment accumulation rate calculated for that interval was determined from the nannofossil $\mathrm{CN} 10 \mathrm{~b}$ and $\mathrm{CN} 10 \mathrm{c}$ Zones found in Section 541-20,CC to Sample $541-22-4,150 \mathrm{~cm}$ (Bergen, this volume). Late Miocene (5-7 Ma) muds and nannofossil muds, drilled from 197 to $276 \mathrm{~m}$ sub-bottom, have dip-corrected bulk sedi- 


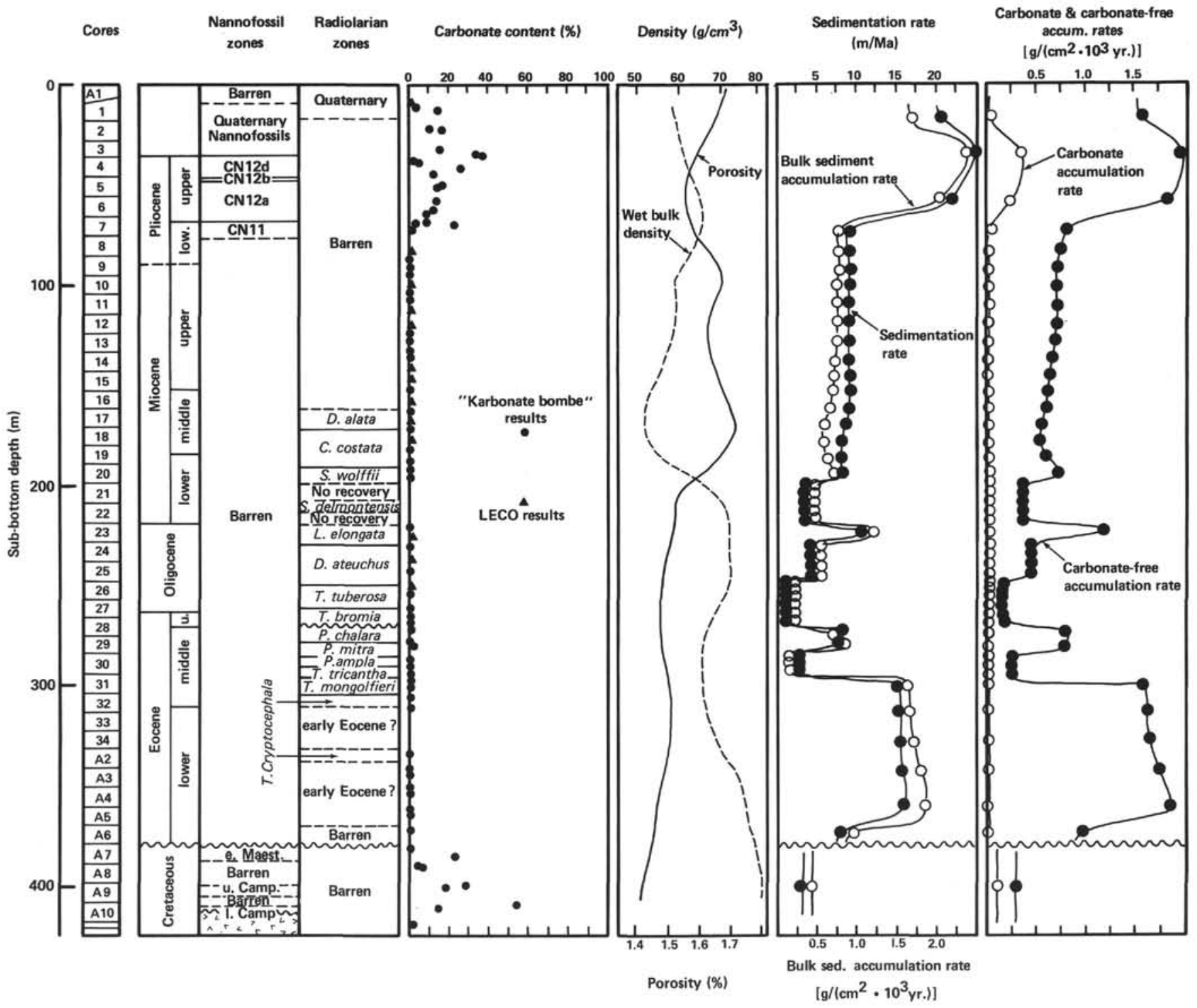

Figure 4. Site 543 nannofossil and radiolarian biostratigraphic zonations, calcium carbonate contents, porosity and wet-bulk density curves, ageversus-depth (sedimentation rate) curve, dip-corrected bulk sediment accumulation rate curve, and carbonate and carbonate-free accumulation rate curve.

ment accumulation rates ranging from 2.6 to $4.2 \mathrm{~g} /\left(\mathrm{cm}^{2}\right.$ - $10^{3}$ yr.) (Table 3). The Miocene 6- to 7-Ma interval contains a repeated nannofossil zone documented by a biostratigraphic inversion between Cores $541-28$ and 541-29. Sediment accumulation rates for that interval were calculated assuming that the boundary between nannofossil Zones $\mathrm{CN} 9 \mathrm{~b}$ and $\mathrm{CN} 9 \mathrm{a}$ is located at the midpoint of the cored interval barren of microfossils from 219 to $248 \mathrm{~m}$ sub-bottom, and that the CN9a nannofossil zone found in Core 541-28 immediately above the biostratigraphic inversion represents the bottom of that zone. Smaller-scale faulting, not documented by any biostratigraphic inversions, may be present but could not be accounted for.

Tectonic Unit B sediments are upper Pliocene to lower Miocene. Pliocene (1.8-5 Ma) nannofossil mudstones occur from 276 to $334 \mathrm{~m}$ sub-bottom and have dip-corrected bulk sediment accumulation rates ranging from
1.8 to $2.8 \mathrm{~g} /\left(\mathrm{cm}^{2} \cdot 10^{3} \mathrm{yr}\right.$.) (Table 3). The late Pliocene rates calculated are minimum rates, because late Pliocene sediments are truncated by the reverse fault at 276 $\mathrm{m}$ sub-bottom that separates Tectonic Unit B from overlying Tectonic Unit A. Miocene mudstones and nannofossil mudstones ranging in age from 5 to $17 \mathrm{Ma}$ were drilled from 334 to $459 \mathrm{~m}$ sub-bottom, at which point drilling was discontinued due to hole collapse. The dipcorrected bulk sediment accumulation rates of these Miocene sediments ranges from 1.6 to $4.4 \mathrm{~g} /\left(\mathrm{cm}^{2} \cdot 10^{3} \mathrm{yr}\right.$. $)$ at 334 to $389 \mathrm{~m}$ sub-bottom, and from 0.6 to $0.7 \mathrm{~g} /\left(\mathrm{cm}^{2}\right.$ - $10^{3} \mathrm{yr}$.) below $389 \mathrm{~m}$ sub-bottom (Table 3 ). The accuracy of these rates is questionable because there are barren intervals between 362 and $376 \mathrm{~m}$ and between 388.5 and $438 \mathrm{~m}$ sub-bottom, but the late Miocene rates were apparently greater than the middle or early Miocene rates calculated for Tectonic Unit B. A latest Miocene sedimentary hiatus is documented by the absence of 
Table 1. Leg 78A "Karbonate Bombe" results.

\begin{tabular}{|c|c|c|c|c|c|}
\hline $\begin{array}{c}\text { Sample } \\
\text { (interval in } \mathrm{cm} \text { ) }\end{array}$ & $\underset{(\%)}{\mathrm{CaCO}_{3}}$ & $\begin{array}{c}\text { Sample } \\
\text { (interval in } \mathrm{cm} \text { ) }\end{array}$ & $\begin{array}{c}\mathrm{CaCO}_{3} \\
(\%)\end{array}$ & $\begin{array}{l}\text { Sample } \\
\text { (interval in } \mathrm{cm} \text { ) }\end{array}$ & $\begin{array}{c}\mathrm{CaCO}_{3} \\
(\%)\end{array}$ \\
\hline $541-1-1,49-51$ & 10.3 & $541-17-2,35-38$ & 34.4 & $541-33-7,31-34$ & 45.4 \\
\hline $541-2-5,11-13$ & 2.5 & $541-17-5,85-88$ & 45.2 & $541-34-2,82-86$ & 33.3 \\
\hline $541-2-5,48-51$ & 7.8 & $541-18-1,108-111$ & 38.8 & $541-34-6,108-112$ & 31.4 \\
\hline $541-2-6,56-58$ & 1.0 & $541-18-3,122-126$ & 41.7 & $541-35-4,103-106$ & 24.2 \\
\hline $541-2-6,71-74$ & 19.6 & $541-19-1,89-92$ & 17.7 & $541-35-6,33-36$ & 38.6 \\
\hline $541-3-1,83-86$ & 4.9 & $541-19-5,64-67$ & 13.7 & $541-36-1,40-45$ & 25.1 \\
\hline $541-3-2,63-64$ & 9.8 & $541-20-1,76-79$ & 37.8 & $541-36-2,29-34$ & 25.1 \\
\hline $541-3-4,68-71$ & 29.8 & $541-20-4,39-43$ & 27.5 & $541-37-1,138-142$ & 4.8 \\
\hline $541-4-1,69-72$ & 37.7 & $541-21-1,72-76$ & 23.1 & $541-37-5,89-93$ & 11.1 \\
\hline $541-4-4,93-96$ & 2.5 & $541-21-2,51-54$ & 30.0 & $541-38-1,84-87$ & 6.0 \\
\hline $541-5-2,7-10$ & 35.7 & $541-22-2,21-24$ & 24.6 & $541-38-7,5-8$ & 0.0 \\
\hline $541-6-1,68-70$ & 9.8 & $541-22-6,91-94$ & 24.1 & $541-39-5,49-52$ & 0.0 \\
\hline $541-7-2,109-112$ & 11.2 & $541-23-1,47-50$ & 27.0 & $541-40-1,40-43$ & 0.5 \\
\hline $541-7-3,53-56$ & 13.7 & $541-23-5,52-55$ & 4.4 & $541-40-4,65-68$ & 1.0 \\
\hline $541-8-1,69-74$ & 28.4 & $541-24-1,69-72$ & 3.4 & $541-41-3,90-95$ & 1.0 \\
\hline $541-8-3,76-78$ & 22.0 & $541-24-4,66-69$ & 1.5 & $541-41-4,36-39$ & 24.4 \\
\hline $541-8-5,42-46$ & 16.6 & $541-25-1,101-104$ & 1.5 & $541-42-1,12-16$ & 1.0 \\
\hline $541-8-7,49-53$ & 23.0 & $541-25-3,54-57$ & 1.5 & $541-42-4,72-78$ & 16.4 \\
\hline $541-9-6,89-91$ & 37.2 & $541-25-7,28-31$ & 0.0 & $541-43-1,107-112$ & 2.0 \\
\hline $541-10-2,79-89$ & 46.9 & $541-26-2,78-80$ & 0.0 & $541-43-4,18-24$ & 0.5 \\
\hline $541-10-4,50-53$ & 31.8 & $541-26-4,68-70$ & 1.0 & $541-43-5,99-103$ & 1.0 \\
\hline $541-10-4,100-102$ & 32.3 & $541-27-1,107-110$ & 0.5 & $541-44-4,123-128$ & 2.0 \\
\hline $541-11-1,99-102$ & 36.7 & $541-27-5,60-63$ & 0.5 & $541-44-7,17-20$ & 1.0 \\
\hline $541-11-4,54-57$ & 35.7 & $541-28-1,90-93$ & 4.8 & $541-45-1,136-140$ & 0.5 \\
\hline $541-12-1,118-121$ & 27.3 & $541-28-4,34-37$ & 6.3 & $541-45-3,42-48$ & 0.5 \\
\hline $541-12-7,12-15$ & 46.0 & $541-28-5,108-111$ & 12.6 & $541-47-1,32-36$ & 0.5 \\
\hline $541-13-1,89-92$ & 40.1 & $541-29-2,30-33$ & 0.5 & $541-47-2,131-135$ & 0.0 \\
\hline $541-13-5,41-44$ & 48.4 & $541-29-4,110-113$ & 2.4 & $541-48-1,105-109$ & 0.5 \\
\hline $541-13-7,25-28$ & 40.6 & $541-30-2,75-79$ & 13.0 & $541-48-3,63-69$ & 0.0 \\
\hline $541-14-3,40-43$ & 35.2 & $541-30-5,103-107$ & 9.2 & $541-48-6,12 \mathrm{i}-125$ & 0.0 \\
\hline $541-14-5,70-73$ & 34.2 & $54 \mathrm{~L}-31-1,130-133$ & 29.0 & $541-49-1,84-87$ & 0.0 \\
\hline $541-15-1,80-83$ & 31.4 & $541-31-6,111-114$ & 39.6 & $541-49-4,2-5$ & 0.5 \\
\hline $541-15-5,41-44$ & 30.4 & $541-32-1,52-55$ & 41.1 & $541-50-1,67-70$ & 0.0 \\
\hline $541-16-3,58-60$ & 28.5 & $541-32-5,34-37$ & 38.2 & & \\
\hline $541-16-6,50-52$ & 41.2 & $541-33-1,104-107$ & 26.6 & & \\
\hline $542-\mathrm{Hl}-2,46-69$ & 40.0 & $542-4-5,81-86$ & 12.0 & $542 \mathrm{~A}-4-1,141-144$ & 9.0 \\
\hline $542-\mathrm{H} 2-2,80-83$ & 29.5 & 542A-H1-2, 64-67 & 27.5 & $542 \mathrm{~A}-4-2,26-30$ & 4.5 \\
\hline $542-\mathrm{H} 2-4,80-83$ & 34.5 & $542 \mathrm{~A}-\mathrm{H} 1-5,84-87$ & 36.5 & $542 A-5-3,108-112$ & 1.0 \\
\hline $542-\mathrm{H} 3-2,69-72$ & 30.5 & 542A-H2-1, 101-104 & 34.0 & $542 \mathrm{~A}-5-5,29-33$ & 22.0 \\
\hline $542-\mathrm{H} 3-3,37-47$ & 28.5 & $542 \mathrm{~A}-\mathrm{H} 2-5,127-130$ & 37.5 & $542 \mathrm{~A}-6-4,97-101$ & 2.5 \\
\hline $542-1-1,99-103$ & 35.5 & $542 \mathrm{~A}-1-2,60-63$ & 27.0 & $542 \mathrm{~A}-6-6,58-62$ & 2.0 \\
\hline $542-1-4,71-75$ & 35.0 & $542 A-1-4,133-136$ & 22.0 & $542 \mathrm{~A}-7-1,132-135$ & 0.5 \\
\hline $542-2-2,112-115$ & 19.5 & $542 \mathrm{~A}-2-1,118-122$ & 0.5 & $542 \mathrm{~A}-7-2,12-15$ & 0.5 \\
\hline $542-2-6,62-66$ & 12.0 & $542 \mathrm{~A}-2-3,90-91$ & 0.5 & $542 \mathrm{~A}-8-1,119-123$ & 11.5 \\
\hline $542-3-1,103-107$ & 21.0 & $542 \mathrm{~A}-2-7,43-47$ & 1.5 & $542 \mathrm{~A}-8-2,8-12$ & 0.0 \\
\hline $542-3-5,98-101$ & 6.5 & $542 \mathrm{~A}-3-3,36-40$ & 0.0 & $542 A-9-3,29-32$ & 10.0 \\
\hline $542-41,55-59$ & 17.5 & $542 A-3-5,106-109$ & 1.0 & & \\
\hline $543-1-2,56-59$ & 0.5 & $543-10-2,82-86$ & 0.0 & $543-27-3,120-123$ & 0.0 \\
\hline $543-1-3,55-56$ & 3.4 & $543-10-4,81-84$ & 0.0 & $543-27-5,83-86$ & 0.0 \\
\hline $543-1-4,111-118$ & 1.0 & $543-11-1,119-123$ & 0.0 & $543-28-1,71-74$ & 0.0 \\
\hline $543-1-7,90-93$ & 14.9 & $543-11-2,90-92$ & 0.0 & $543-28-3,59-62$ & 0.0 \\
\hline $543-2-3,44-47$ & 10.4 & $543-11-3,59-63$ & 0.0 & $543-29-2,56-60$ & 0.0 \\
\hline $543-2-3,58-59$ & 16.8 & $543-12-2,117-121$ & 0.0 & $543-29-3,61-65$ & 1.0 \\
\hline $543-2-5,44-47$ & 0.0 & $543-12-4,70-72$ & 0.0 & $543-30-2,110-113$ & 0.0 \\
\hline $543-3-3,120-123$ & 15.3 & $543-12-6,125-129$ & 0.0 & $543-30-5,125-128$ & 0.0 \\
\hline $543-3-5,40-43$ & 34.2 & $543-13-2,116-120$ & 0.0 & $543-30-6,82-88$ & 0.0 \\
\hline $543-3-5,56-57$ & 37.6 & $543-13-6,70-74$ & 0.0 & $543-31-1,77-81$ & 0.0 \\
\hline $543-4-2,60-63$ & 2.5 & $543-14-2,80-83$ & 0.0 & $543-31-2,8-11$ & 0.0 \\
\hline $543-4-2,89-91$ & 5.0 & $543-14-5,110-113$ & 0.0 & $543-32-1,81-84$ & 0.0 \\
\hline $543-4-4,60-63$ & 25.2 & $543-15, C C$ & 0.0 & $543-32-4,43-47$ & 0.0 \\
\hline $543-4-6,130-133$ & 11.9 & $543-16-2,92-95$ & 0.0 & $543 \mathrm{~A}-2-2,52-55$ & 0.0 \\
\hline $543-5-3,13-16$ & 15.3 & $543-16-4,23-27$ & 0.0 & $543 \mathrm{~A} \cdot 2, \mathrm{CC}$ & 0.0 \\
\hline $543-5-3,81-82$ & 14.3 & $543-17-1,70-73$ & 0.0 & $543 \mathrm{~A}-3-1,148-150$ & 0.0 \\
\hline $543-6-2,135-137$ & 13.8 & $543-17-4,135-138$ & 0.0 & $543 \mathrm{~A}-3-2,101-104$ & 0.0 \\
\hline $543-6-4,70-72$ & 12.3 & $543-18-1,65-68$ & 0.0 & $543 A-4-1,6-9$ & 0.0 \\
\hline $543-6-6,70-72$ & 9.9 & $543-18-6,99-102$ & 0.0 & $543 A-4-3,36-39$ & 0.0 \\
\hline $543-6-7,21-23$ & 8.4 & $543-19-3,80-84$ & 0.0 & $543 \mathrm{~A}-5-\mathrm{a}, 84-87$ & 0.0 \\
\hline $543-7-2,94-97$ & 9.4 & $543-19-3,93-97$ & 0.0 & $543 \mathrm{~A}-5-2,58-61$ & 0.0 \\
\hline $543-7-3,74-76$ & 3.9 & $543-19-6,13-16$ & 0.0 & $543 \mathrm{~A}-6-1,49-52$ & 0.0 \\
\hline $543-7-3,92-94$ & 22.2 & $543-20-2,98-101$ & 0.0 & $543 \mathrm{~A}-7-1,96-100$ & 0.0 \\
\hline $543-7-4,41-44$ & 2.0 & $543-20-3,88-92$ & 0.0 & $543 \mathrm{~A}-7-3,100-104$ & 24.4 \\
\hline $543-8-3,116-118$ & 0.0 & $543-23-1,138-142$ & 0.0 & $543 \mathrm{~A}-8-1,17-20$ & 4.9 \\
\hline $543-8-3,124-128$ & 0.0 & $543-23-2,99-103$ & 0.0 & $543 \mathrm{~A}-8-1,87-91$ & 5.4 \\
\hline $543-8-4,50-54$ & 0.0 & $543-24-2,108-111$ & 0.0 & $543 \mathrm{~A}-9-1,6-10$ & 17.6 \\
\hline $543-8, C C, 10-13$ & 0.0 & $543-24-4,103-106$ & 0.0 & $543 A-9-1,50-54$ & 28.8 \\
\hline $543-9-1,104-107$ & 0.0 & $543-25-1,11-15$ & 0.0 & $543 \mathrm{~A}-10-1,28-32$ & 53.2 \\
\hline $543-9-3,84-86$ & 0.0 & $543-25-2,55-59$ & 0.0 & $543 \mathrm{~A}-10-1,120-124$ & 14.2 \\
\hline $543-9-4,65-68$ & 0.5 & $543-26-2,54-57$ & 0.0 & $543 \mathrm{~A}-11-2,30-34$ & 1.0 \\
\hline $543-10-1,60-62$ & 0.0 & $543-26-5,55-58$ & 0.0 & & \\
\hline
\end{tabular}

nannofossil CN10a Zone sediments drilled in Tectonic Unit B. The CN10a Zone drilled in overlying Tectonic Unit A (Core 541-22) is correspondingly very thin.

Carbonate percentages range from 5 to $35 \%$ in the Quaternary to upper Miocene sediments cored in both Tectonic Units A and B (Tables 1 and 2), indicating 1Ma-increment carbonate accumulation rates of 0.1 to $1.8 \mathrm{~g} /\left(\mathrm{cm}^{2} \cdot 10^{3} \mathrm{yr}\right.$ ) (Fig. 2; Table 3). The Miocene sediments of Tectonic Unit B below $348 \mathrm{~m}$ sub-bottom have
Table 2. Leg 78A LECO Carbon Analyzer results.

\begin{tabular}{|c|c|c|c|c|c|c|c|}
\hline $\begin{array}{l}\text { Sample } \\
\text { (cm level) }\end{array}$ & $\begin{array}{c}\text { Total } \\
\text { carbon }\end{array}$ & $\begin{array}{l}\text { Organic } \\
\text { carbon }\end{array}$ & $\begin{array}{c}\mathrm{CaCO}_{3} \\
(\%)\end{array}$ & $\begin{array}{c}\text { Sample } \\
\text { (cm level) }\end{array}$ & $\begin{array}{c}\text { Total } \\
\text { carbon }\end{array}$ & $\begin{array}{c}\text { Organic } \\
\text { carbon }\end{array}$ & $\begin{array}{c}\mathrm{CaCO}_{3} \\
(\%)\end{array}$ \\
\hline $541-3-3,73$ & 1.5 & 0.2 & 11 & $541-20-4,103$ & 4.0 & 0.1 & 33 \\
\hline $541-4-4,100$ & 0.7 & 0.2 & 4 & $541-21-2,120$ & 3.0 & 0.1 & 25 \\
\hline $541-5-1,73$ & 0.4 & 0.1 & 2 & $541-22-2,58$ & 3.9 & 0.1 & 32 \\
\hline $541-5-2,80$ & 0.3 & 0.1 & 1 & $541-25-3,60$ & 0.1 & 0.1 & 0 \\
\hline $541-6-1,80$ & 0.6 & 0.2 & 3 & $541-27-5,55$ & 0.2 & 0.1 & 1 \\
\hline $541-7-4,100$ & 0.8 & 0.2 & 5 & $541-28-2,83$ & 0.2 & 0.1 & 1 \\
\hline $541-8-4,70$ & 4.7 & 0.2 & 38 & $541-29-2,110$ & 0.1 & 0.1 & 1 \\
\hline $541-9-5,110$ & 4.6 & 0.2 & 37 & $541-29-4,94$ & 1.1 & 0.1 & 9 \\
\hline $541-11-4,62$ & 4.6 & 0.2 & 37 & $541-30-4,26$ & 1.1 & 0.1 & 9 \\
\hline $541-12-1,116$ & 3.5 & 0.1 & 28 & $541-30-7,25$ & 5.3 & 0.1 & 43 \\
\hline $541-13-1,60$ & 4.8 & 0.2 & 39 & $541-31-3,20$ & 4.9 & 0.1 & 40 \\
\hline $541-14-6,60$ & 4.4 & 0.1 & 36 & $541-33-5,88$ & 5.9 & 0.1 & 49 \\
\hline $541-15-1,90$ & 3.9 & 0.2 & 31 & $541-34-2,135$ & 4.5 & 0.1 & 37 \\
\hline $541-16-4,120$ & 3.6 & 0.1 & 29 & $541-38-1,16$ & 1.2 & 0.1 & 9 \\
\hline $541-17-5,90$ & 5.5 & 0.1 & 45 & $541-40-3,71$ & 0.1 & 0.1 & 0 \\
\hline $541-18-3,86$ & 3.9 & 0.1 & 31 & $541-41-1,76$ & 1.6 & 0.1 & 13 \\
\hline $541-19-1,71$ & 3.0 & 0.1 & 24 & $541-44-2,49$ & 0.3 & 0.1 & 2 \\
\hline $542-\mathrm{H} 3-2,30$ & 5.4 & 0.1 & 45 & $542 \mathrm{~A}-2-3,85$ & 0.5 & 0.1 & 4 \\
\hline $542-1-2,62$ & 4.3 & 0.1 & 35 & $542 \mathrm{~A}-4-2,101$ & 1.7 & 0.1 & 13 \\
\hline $542-2-2,106$ & 3.1 & 0.0 & 25 & $542 \mathrm{~A}-5-5,60$ & 2.4 & 0.1 & 20 \\
\hline $542-3-3,30$ & 3.7 & 0.1 & 30 & $542 \mathrm{~A}-6-2,92$ & 0.2 & 0.1 & 1 \\
\hline $542-4-3,145$ & 0.7 & 0.1 & 6 & $542 \mathrm{~A}-9-1,100$ & 1.6 & 0.1 & 13 \\
\hline $543-1-3,60$ & 0.2 & 0.1 & 0 & $543-16-3,50$ & 0.1 & 0.1 & 0 \\
\hline $543-7-3,34$ & 0.3 & 0.1 & 2 & $543-17-2,77$ & 0.1 & 0.1 & 0 \\
\hline $543-8-4,60$ & 0.3 & 0.1 & 2 & $543-18-3,10$ & 0.1 & 0.1 & 0 \\
\hline $543-10-3,60$ & 0.1 & 0.1 & 0 & $543-18-4,43$ & 0.2 & 0.1 & 1 \\
\hline $543-11-3,90$ & 0.1 & 0.1 & 0 & $543-23-2,131$ & 0.1 & 0.1 & 0 \\
\hline $543-12-1,80$ & 0.1 & 0.1 & 0 & $543-24-4,28$ & 0.1 & 0.1 & 0 \\
\hline $543-14-4,83$ & 0.1 & 0.1 & 0 & $543-25-2,99$ & 0.1 & 0.1 & 0 \\
\hline $543-15-2,111$ & 0.1 & 0.1 & $\mathbf{0}$ & $543-26-3,57$ & 0.1 & 0.1 & 0 \\
\hline
\end{tabular}

no carbonate component, and the carbonate accumulation rates of those sediments is therefore zero.

\section{Site $\mathbf{5 4 2}$}

Site 542 was drilled in the Lesser Antilles lower slope environment, $1.5 \mathrm{~km}$ landward of the deformation front (Fig. 1). Three holes were drilled at Site 542: Hole 542 was interval cored above $202 \mathrm{~m}$ and continuously cored from 202 to $240 \mathrm{~m}$ sub-bottom, where a $7.6^{\circ}$ deviation from vertical necessitated abandonment of the hole. Hole $542 \mathrm{~A}$ was cored from 173.5 to $183 \mathrm{~m}$ and 240 to $325.5 \mathrm{~m}$ sub-bottom, with interval wash cores taken between 0 and $173.5 \mathrm{~m}$ and 183 and $240 \mathrm{~m}$ sub-bottom. Hole 542 A was abandoned because of hole collapse in a zone of intensely sheared and fractured mudstone. No sediments were recovered from Hole 542B. Interval wash cores are disregarded in sediment accumulation rate calculations due to uncertainty of where within the washed interval the recovered material actually originated (Fig. 3 ; Table 3). Core recoveries in the continuously cored interval ranged from 26 to $105 \%$, averaging $84 \%$. Smallscale biostratigraphic inversions are documented between Cores 542A-1 and 542-1 and between Cores 542A8 and 542A-9 (Fig. 3). Intervals barren of microfossils are found at 240 and $249.5 \mathrm{~m}$ (Core 542A-2) and 263.5 and $270 \mathrm{~m}$ sub-bottom (Sections $542 \mathrm{~A}-4-4$ to $542 \mathrm{~A}-5-1$ ).

Quaternary $(0-1.8 \mathrm{Ma})$ sediments were not continuously cored at Site 542. Estimations of Quaternary dipcorrected bulk sediment accumulation rates were made by assuming the following: (1) that sediment exposed on the seafloor is modern sediment; (2) that the top of the continuously cored interval (Core $542 \mathrm{~A}-1$ at $173.5 \mathrm{~m}$ subbottom) represents the top of nannofossil CN11a Zone (Bukry and Okada, 1980) deposited 3.5 Ma; and (3) that the sedimentation rate from 0 to $3.5 \mathrm{Ma}$ was constant. On the basis of these assumptions, and incorporating porosity and density values from comparable depths at 
Table 3. Sediment accumulation rate data.

\begin{tabular}{|c|c|c|c|c|c|c|c|c|c|c|c|c|}
\hline & $\begin{array}{c}\text { Age } \\
\text { (from Bukry } \\
\text { and Okada, } \\
\text { 1980) } \\
\end{array}$ & $\begin{array}{c}\text { Age } \\
\text { interval } \\
(\mathrm{m} / \mathrm{Ma})\end{array}$ & $\begin{array}{l}\text { Sample } \\
\text { (cm from top of section) }\end{array}$ & $\begin{array}{c}\text { Sedimentation } \\
\text { rate } \\
(\mathrm{m} / \mathrm{Ma})\end{array}$ & $\begin{array}{c}\text { Porosity } \\
(\%)\end{array}$ & $\begin{array}{l}\text { Density } \\
\left(\mathrm{g} / \mathrm{cm}^{3}\right)\end{array}$ & $\begin{array}{c}\text { Bulk sediment } \\
\text { accum. rate } \\
{\left[\mathrm{g} /\left(\mathrm{cm}^{2} \cdot 10^{3} \mathrm{yr} .\right)\right]}\end{array}$ & $\begin{array}{l}\text { Approx. } \\
\text { bed. dip } \\
\text { (०) }\end{array}$ & $\begin{array}{c}\text { Dip-corrected } \\
\text { bulk sed. } \\
\text { accum. arte } \\
{\left[8 /\left(\mathrm{cm}^{2} \cdot 10^{3} \mathrm{yz}\right)\right]}\end{array}$ & $\begin{array}{l}\text { Approx. } \\
\mathrm{CaCO}_{3} \\
(\%)\end{array}$ & $\begin{array}{c}\text { Carbonate } \\
\text { accum. rate } \\
{\left[\mathrm{g} /\left(\mathrm{cm}^{2} \cdot 10^{3} \mathrm{yr}\right)\right]}\end{array}$ & $\begin{array}{c}\text { Carbonate-free } \\
\text { accum. rate } \\
{\left[\mathrm{g} /\left(\mathrm{cm}^{2} \cdot 10^{3} \mathrm{yr} .\right)\right]} \\
\end{array}$ \\
\hline \multirow{41}{*}{$\begin{array}{c}\text { Tetconic } \\
\text { Unit A }\end{array}$} & Quat. & $0-1$ & $541-1-1,0$ to $541-7-2,55$ & 50.55 & 66 & 1.65 & 4.92 & 0 & 4.92 & 10 & 0.49 & 4.43 \\
\hline & $\underline{3}$ & $\begin{array}{l}1-2 \\
2-3\end{array}$ & $\begin{array}{l}5411-7-2,55 \text { to } 544-12,2 C \\
541-13-1,0 \text { to } 541-164,150\end{array}$ & $\begin{array}{l}54.95 \\
34.50\end{array}$ & $\begin{array}{l}61 \\
68\end{array}$ & 1.73 & $\begin{array}{l}6.07 \\
4.06\end{array}$ & 0 & 6.07 & 30 & 1.82 & 4.25 \\
\hline & Pliocene $=$ & $3-4$ & $\begin{array}{l}341-13-1,0,0 \text { to } \\
541-16-5,0 \text { to } \\
441-18-6,6,150\end{array}$ & $\begin{array}{l}32.00 \\
22.00\end{array}$ & $\begin{array}{l}58 \\
56\end{array}$ & $\begin{array}{l}1.77 \\
1.80\end{array}$ & $\begin{array}{l}4.06 \\
2.70\end{array}$ & 20 & $\begin{array}{l}3.81 \\
2.53\end{array}$ & 35 & 1.33 & 2.48 \\
\hline & & $4-5$ & $541-20, C \mathrm{CC}$ to $541-22-4,150$ & 16.00 & 55 & 1.83 & $\begin{array}{l}2.70 \\
2.03\end{array}$ & ${ }_{20}^{20}$ & $\begin{array}{l}2.53 \\
1.91\end{array}$ & 30 & 0.76 & 1.77 \\
\hline & Miocene ${ }^{\prime}$ & $\begin{array}{c}5-6 \\
6-7\end{array}$ & $541-22-5,0$ to $541-24, C C$ & 22.50 & 58 & 1.81 & 2.73 & ${ }_{20}^{20}$ & $\begin{array}{l}1.91 \\
2.57\end{array}$ & 25 & $\begin{array}{l}0.48 \\
0.13\end{array}$ & 1.43 \\
\hline & Mivene & $6-7$ & $541-25-1,0$ to $541-28, \mathrm{CC}$ & 38.00 & 59 & 1.78 & 4.46 & 20 & $\begin{array}{l}2.37 \\
4.19\end{array}$ & ${ }_{5}^{5}$ & 0.21 & $\begin{array}{l}2.44 \\
3.98\end{array}$ \\
\hline & Pliocene & $\begin{array}{c}2-3 \\
3-4\end{array}$ & $\begin{array}{l}541-30-6,130 \text { to } 514-333-2,1,10 \\
541-33-3,0 \text { to } 541-35-3,105\end{array}$ & $\begin{array}{l}22.70 \\
20.50\end{array}$ & $s 1$ & 1.87 & 3.06 & 25 & 2.77 & 25 & 0.69 & 2.08 \\
\hline & Plocene & 4-5 & S41-35-4, o to $541-36, \mathrm{CC}$ & $\begin{array}{l}20.50 \\
14.50\end{array}$ & $\begin{array}{l}51 \\
52\end{array}$ & 1.90 & 2.82 & 25 & 2.56 & 30 & 0.77 & 1.79 \\
\hline & & $5-7$ & $541-37-1,0$ to $541-38-4,20$ & $\begin{array}{l}14.30 \\
14.20\end{array}$ & $\begin{array}{l}52 \\
55\end{array}$ & $\begin{array}{l}1.87 \\
1.79\end{array}$ & $\begin{array}{l}1.94 \\
1.79\end{array}$ & 25 & 1.76 & 25 & 0.44 & 1.32 \\
\hline & & $6-7$ & $541-38-4,20$ to $541-42-5,150$ & 40.80 & 57 & 1.77 & 4.84 & ${ }_{25}^{25}$ & $\begin{array}{l}1.58 \\
4.38\end{array}$ & $\begin{array}{c}10 \\
0\end{array}$ & $\begin{array}{l}0.16 \\
0\end{array}$ & $\begin{array}{l}1.42 \\
4.38\end{array}$ \\
\hline & & $7-8$ & $541-42-6,0$ to $541-43-4,10$ & & & 1.73 & 0.73 & 25 & 0.66 & 0 & 0 & $\begin{array}{l}4.38 \\
0.66\end{array}$ \\
\hline & 育 & $\begin{array}{l}89-9 \\
9-10\end{array}$ & $541-43-4,10$ to $541-44-2,20$ & & 63 & 1.72 & 0.71 & 25 & 0.64 & 0 & 0 & 0.64 \\
\hline & Miocene & $\begin{array}{l}9-10 \\
10-11\end{array}$ & $541-44-2,20$ to $541-44-6,80$ & 6.60 & 64 & 1.71 & 0.70 & 25 & 0.63 & 0 & 0 & 0.63 \\
\hline & Miocene & 11-12 & $541-44-6,80$ to $541-45-4,90$ & 6.60 & 63 & 1.70 & 0.70 & 25 & 0.63 & 0 & 0 & 0.63 \\
\hline & & $\int_{12-13}^{11-12}$ & $\begin{array}{l}541-45-4,90 \text { to } 541-46-2,100 \\
541-46-2,100 \text { } 541-46-7,10\end{array}$ & 6.60 & 62 & 1.70 & 0.70 & 25 & 0.64 & 0 & 0 & 0.64 \\
\hline & & $13-14$ & $\begin{array}{l}544-46-2,100 \text { to } 541-46-7,10 \\
541-46-7,100541-47.5,20\end{array}$ & 6.60 & 61 & 1.70 & 0.71 & 25 & 0.64 & 0 & 0 & 0.64 \\
\hline & E & $14-15$ & $\begin{array}{l}541-46-7,10 \text { to } 541-47-5,20 \\
541-77-5,20 \text { to } 541-48-3,70\end{array}$ & 6.60 & 61 & 1.70 & 0.71 & ${ }_{-25}^{25}$ & 0.64 & 0 & 0 & 0.64 \\
\hline & & $15-16$ & $541-48-3,70$ to $541-49-2,10$ & 7.00 & $\begin{array}{l}60 \\
60\end{array}$ & 1.70 & $\begin{array}{l}0.76 \\
0.80\end{array}-10$ & ${ }_{25}^{25}$ & $\begin{array}{l}0.69 \\
0.73\end{array}$ & $\begin{array}{l}0 \\
0\end{array}$ & $\begin{array}{l}0 \\
0\end{array}$ & $\begin{array}{l}0.69 \\
0.73\end{array}$ \\
\hline & 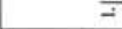 & $16-17$ & $541-49-2,10$ to $541-50-1,150$ & 7.40 & 59 & 1.70 & 0.81 & 25 & 0.73 & 0 & 0 & 0.73 \\
\hline & Quat. & $0-1$ & & 49.60 & 66 & 1.65 & 4.83 & 16 & 4.64 & 30 & 1.39 & 3.25 \\
\hline & 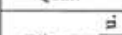 & ${ }_{2-3}^{1-2}$ & Not continuously cored & 49.60 & 61 & 1.73 & 5.48 & 16 & 5.27 & 30 & 1.58 & 3.69 \\
\hline & Pliocene $=$ & $3-4$ & $542 A-1-1,0$ to $542 A-1-4,150$ & $\begin{array}{l}49.60 \\
30.80\end{array}$ & 56 & $\begin{array}{l}1.77 \\
1.79\end{array}$ & 3.74 & $\begin{array}{l}16 \\
16\end{array}$ & $\begin{array}{l}5.60 \\
3.60\end{array}$ & 30 & $\begin{array}{l}1.08 \\
1.08\end{array}$ & $\begin{array}{l}3.92 \\
2.52\end{array}$ \\
\hline & & $4-5$ & $542-2-1,0$ to $542-3-3,150$ & 14.00 & 53 & 1.78 & 1.73 & 16 & 1.66 & 20 & 0.83 & 1.33 \\
\hline & Miocene & $\begin{array}{c}3-6 \\
6 \rightarrow 7\end{array}$ & 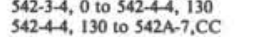 & $\begin{array}{l}10.70 \\
60.80\end{array}$ & $\begin{array}{l}54 \\
58\end{array}$ & 1,78 & 1.31 & ${ }_{17}^{16}$ & 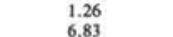 & 15 & $\begin{array}{l}0.19 \\
0.34\end{array}$ & $\begin{array}{l}1.07 \\
6.49\end{array}$ \\
\hline & & & $543 \mathrm{~A}-1-1,0$ to $543-2.2,50$ & & & & .1. & 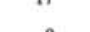 & & 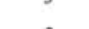 & & 6.49 \\
\hline & Quat. & $1-2$ & $543-2-2,50$ to $543-4, C C$ & 26.5 & 65 & $\begin{array}{l}1.53 \\
1.56\end{array}$ & $\begin{array}{l}1.37 \\
2.37\end{array}$ & ${ }_{0}^{0}$ & $\begin{array}{l}1.66 \\
2.37\end{array}$ & ${ }_{15}^{2}$ & $\begin{array}{l}0.033 \\
0.36\end{array}$ & $\begin{array}{l}1.63 \\
2.01\end{array}$ \\
\hline & Pliopene $\stackrel{i}{=}$ & $\begin{array}{l}2-3 \\
3-4\end{array}$ & $543-5-1,0$ to $543-7-2,150$ & 22.0 & 63 & 1.60 & 2.10 & 0 & 2.10 & 12 & 0.25 & 1.85 \\
\hline & & $\begin{array}{l}3-4 \\
4-5\end{array}$ & $543-8-2,100$ to $543-9-2,45$ & 8.95 & $\begin{array}{l}65 \\
67\end{array}$ & 1.59 & 0.83 & 0 & 0.83 & 3 & 0.03 & 0.80 \\
\hline & & $5-6$ & $543-9-2,45$ to $543-10-1,135$ & 8.95 & 70 & $\begin{array}{l}1.56 \\
1.53\end{array}$ & $\begin{array}{l}0.78 \\
0.73\end{array}$ & 0 & $\begin{array}{l}0.78 \\
0.73\end{array}$ & & $0_{0}^{0}$ & 0.78 \\
\hline & & $6-7$ & $543-10-1,135$ to $543-11-1,80$ & 8.95 & 70 & 1.52 & 0.72 & 0 & 0.72 & 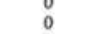 & 0 & $\begin{array}{l}0.72 \\
0.72\end{array}$ \\
\hline & $\bar{g}$ & $7-8$ & $543-11-1,80$ to $543-12-1,25$ & 8.95 & 69 & 1.53 & 0.74 & 0 & 0.74 & 0 & 0 & 0.74 \\
\hline & है & $\begin{array}{l}89 \\
9-10\end{array}$ & $\begin{array}{l}543-12-1,25 \text { to } 543-12, \mathrm{CC} \\
543-12 \mathrm{CC} \text { to } 543-13-6,150\end{array}$ & $\begin{array}{l}8.95 \\
8.95\end{array}$ & $\begin{array}{l}69 \\
69\end{array}$ & 1.53 & 0.74 & 0 & 0.74 & 0 & 0 & 0.74 \\
\hline & & $10-11$ & $543-13-7,0$ to $543-14-6,65$ & 8.95 & ${ }_{70}^{69}-x-1$ & $\begin{array}{l}1.51 \\
1.48\end{array}$ & $\begin{array}{l}0.72 \\
0.68\end{array}$ & ${ }_{0}^{0}$ & $\begin{array}{l}0.72 \\
0.68\end{array}$ & $\begin{array}{l}0 \\
0\end{array}$ & $\begin{array}{l}0 \\
0\end{array}$ & 0.68 \\
\hline & & $11-12$ & $543-14-6,65$ to $543-15-6,10$ & 8.95 & 71 & 1.46 & 0.66 & 0 & 0.66 & 0 & 0 & $\begin{array}{l}0.68 \\
0.66\end{array}$ \\
\hline & & $12-13$ & $543-15-6,10$ to $543-16-5,105$ & 8.95 & 72 & 1.44 & 0.63 & 0 & 0.63 & 0 & 0 & 0.63 \\
\hline & Miocene $\mathrm{E}$ & $13-14$ & $543-16-5,105$ to $543-17-5,50$ & 8.95 & 73 & 1.43 & 0.61 & 0 & 0.61 & 0 & 0 & 0.61 \\
\hline & miverie of & $\mid \begin{array}{l}14-15 \\
15-16\end{array}$ & $\begin{array}{l}543-17-5,50 \text { to } 543-18-4,80 \\
543-18-4,80 \text { t } 543-19-3,40\end{array}$ & $\begin{array}{l}8.30 \\
7.60\end{array}$ & 73 & 1.43 & 0.56 & 0 & 0.56 & 0 & 0 & $\begin{array}{l}0.56 \\
0.56\end{array}$ \\
\hline & & $16-17$ & $\begin{array}{l}5434-18-4,80 \text { to } 543-19-1-3,40 \\
543-19-3,40 \text { to } 543-20-1,150\end{array}$ & 7,60 & $\begin{array}{l}71 \\
67\end{array}$ & $\begin{array}{l}1.46 \\
1.50\end{array}$ & $\begin{array}{l}0.56 \\
0.62\end{array}$ & $\stackrel{0}{0}$ & $\begin{array}{l}0.56 \\
0.62\end{array}$ & $\begin{array}{l}0 \\
0\end{array}$ & $\begin{array}{l}0 \\
0\end{array}$ & $\begin{array}{l}0.36 \\
0.62\end{array}-10$ \\
\hline & & $17-18$ & $543-20-2$ to $543-20, \mathrm{CC}$ & 8.00 & 63 & 1.57 & 0.74 & 0 & 0.74 & 0 & $0_{0}^{0}$ & $\begin{array}{l}0.74 \\
0.72\end{array}-12$ \\
\hline & & $18-19$ & $543-21-1,0$ to $543-21-3,80$ & 3.80 & 61 & 1.61 & 0.37 & 0 & 0.37 & 0 & 0 & 0.37 \\
\hline & $\underline{\underline{g}}$ & $19-20$ & $543-21-3,80$ to $543-21-6,10$ & 3.80 & 60 & 1.63 & 0.38 & 0 & 0.38 & 0 & 0 & 0.38 \\
\hline \multirow{39}{*}{ Unit B } & & $20-21$ & $543-21-6,10$ to $543-22-2,40$ & 3.80 & 59 & 1.65 & 0.40 & 0 & 0.40 & 0 & 0 & 0.40 \\
\hline & & $\begin{array}{l}221-22 \\
22-23\end{array}$ & 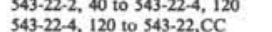 & $\begin{array}{l}3.80 \\
3.80\end{array}$ & 59 & 1.66 & 0.40 & 0 & 0.40 & 0 & 0 & 0.40 \\
\hline & & $23-24$ & $\begin{array}{l}3433-23-4,120 \\
543-23-1,0 \text { to } 543-24-1,1,150\end{array}$ & $\begin{array}{r}31.80 \\
11.00\end{array}$ & $\begin{array}{l}59 \\
59\end{array}$ & $\begin{array}{l}1.67 \\
1.68\end{array}$ & $\begin{array}{l}0.40 \\
1.18\end{array}$ & $\begin{array}{c}0 \\
0\end{array}$ & $\begin{array}{l}0.40 \\
1.8\end{array}$ & $\begin{array}{l}0 \\
0\end{array}$ & ${ }_{0}^{0}$ & 0.40 \\
\hline & & $24-25$ & $543-24-2,0$ to $543-24-5,10$ & 4.60 & 58 & $\begin{array}{l}1.68 \\
1.68\end{array}$ & $\begin{array}{l}1.18 \\
0.50\end{array}$ & ${ }_{0}^{0}$ & $\begin{array}{l}1.18 \\
0.50\end{array}$ & $\begin{array}{l}0 \\
0\end{array}$ & $\begin{array}{l}0 \\
0\end{array}$ & $\begin{array}{l}1.18 \\
0.50\end{array}$ \\
\hline & & $25-26$ & $543-24-5,10$ to $543-25-1,120$ & 4.60 & 57 & 1.68 & 0.50 & 0 & 0.50 & 0 & 0 & $\begin{array}{l}0.90 \\
0.50\end{array}$ \\
\hline & 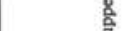 & ${ }_{\gamma \rightarrow-2}^{26-27}$ & $543-25-1,120$ to $543-25-4,130$ & 4.60 & 57 & 1.68 & 0.50 & 0 & 0.50 & 0 & 0 & 0.50 \\
\hline & $\vec{z}$ & ${ }_{28}^{27-28}$ & & 4.60 & 57 & 1.68 & 0.50 & 0 & 0.50 & 0 & 0 & 0.50 \\
\hline & & 29-30 & $\begin{array}{l}543-26-1,90 \text { to } 5433-26-3,20 \\
543-26-3,20 \text { to } 543-26-4,20\end{array}$ & $\begin{array}{l}2.30 \\
1.53\end{array}$ & $\begin{array}{l}57 \\
57\end{array}$ & $\begin{array}{l}1.68 \\
1.68\end{array}$ & 0.25 & ${ }_{0}^{0}$ & $\begin{array}{l}0.25 \\
0.17\end{array}$ & $\begin{array}{l}0 \\
0\end{array}$ & 0 & 0.25 \\
\hline & Oligocene_ & $30-31$ & $543-26-4,20$ to $543-26-5,20$ & 1.53 & 37 & $\begin{array}{l}1.68 \\
1.68\end{array}$ & $\begin{array}{l}0.17 \\
0.17\end{array}$ & 0 & $\begin{array}{l}0.17 \\
0.17\end{array}$ & 0 & $\begin{array}{l}0 \\
0\end{array}$ & 0.17 \\
\hline & & $31-32$ & $543-26-5,20$ to $543-26-6,20$ & 1.53 & 57 & 1.68 & 0.17 & 0 & 0.17 & 0 & 0 & $\begin{array}{l}0.17 \\
0.17\end{array}$ \\
\hline & & $32-33$ & $543-26-6,20$ to $543-26, \mathrm{CC}$ & 1.53 & 57 & 1.68 & 0.17 & 0 & 0.17 & 0 & 0 & 0.17 \\
\hline & 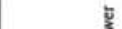 & 33-34 & $543-26, \mathrm{CC}$ to $543-27-1,120$ & 1.53 & 57 & 1.67 & 0.17 & 0 & 0.17 & 0 & 0 & 0.17 \\
\hline & $\underline{\underline{g}}$ & 34-35 & $543-27-1,120$ to $543-27-2,120$ & 1.53 & 57 & 1.67 & 0.17 & 0 & 0.17 & 0 & 0 & 0.17 \\
\hline & & $35-36$ & $543-27-2,120$ to $543-27-3,140$ & 1.70 & 57 & 1.66 & 0.18 & 0 & 0.18 & 0 & 0 & 0.18 \\
\hline & & 36-37 & $543-27-3,140$ to $543-27-4,140$ & 1.53 & 57 & 1.66 & 0.16 & 0 & 0.16 & 0 & 0 & 0.16 \\
\hline & & $\begin{array}{l}37-38 \\
38-39 \\
38\end{array}-10$ & $543-27-4,140$ to $543-27-5,140$ & 1.53 & 57 & 1.66 & 0.16 & 0 & 0.16 & 0 & 0 & 0.16 \\
\hline & ğ & 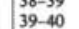 & 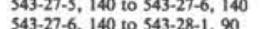 & 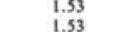 & 57 & 1.65 & 0.16 & ${ }_{0}^{0}$ & 0.16 & & $0_{0}^{0}$ & 0.16 \\
\hline & E & $40-41$ & $543-28-1,90$ to $543-28-2,90$ & 1.53 & 57 & $\begin{array}{l}1.165 \\
1.64\end{array}$ & $\begin{array}{l}0.16 \\
0.16\end{array}$ & $\begin{array}{l}0 \\
0\end{array}$ & $\begin{array}{l}0.16 \\
0.16\end{array}$ & ${ }_{0}^{0}$ & 0 & $\begin{array}{l}0.16 \\
0.16\end{array}$ \\
\hline & & $41-42$ & 543-28-2, 90 to $543-28-3,90$ & 1.53 & 57 & 1.64 & 0.16 & 0 & 0.16 & 0 & 0 & 0.16 \\
\hline & & $42-43$ & $543-28-3,90$ to $543-28-3,150$ & 0.60 & 57 & 1.63 & 0.06 & 0 & 0.06 & 0 & 0 & 0.06 \\
\hline & & $43-44$ & $543-28-4,0$ to $543-29-2,150$ & 8.00 & 57 & 1.62 & 0.83 & 0 & 0.83 & 0 & 0 & 0.83 \\
\hline & & $44-45$ & $543-29-3,0$ to $543-30-1,100$ & 7.50 & 57 & 1.62 & 0.78 & 0 & 0.78 & 0 & 0 & 0.78 \\
\hline & Eocene 券 & $\begin{array}{l}45-46 \\
46-47\end{array}$ & $543-30-1,100$ to $543-30-3,30$ & 2.333 2.33 & 57 & 1.62 & 0.24 & 0 & 0.24 & 0 & 0 & \\
\hline & & 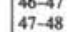 & $\begin{array}{l}543-30-30-4,115 \text { to } 543-30-6,5,50 \\
543-30\end{array}$ & $\begin{array}{l}2.33 \\
2.34\end{array}$ & $\begin{array}{c}57 \\
57\end{array}$ & $\begin{array}{l}1.62 \\
1.62\end{array}$ & $\begin{array}{l}0.24 \\
0.24\end{array}$ & 0 & 0.24 & & ${ }_{0}^{0}$ & $\begin{array}{l}0.24 \\
0.24\end{array}$ \\
\hline & & $\begin{array}{l}48-49 \\
48-78\end{array}-10$ & $543-30-6$, s0 to $543-32-4,30$ & $\begin{array}{l}2.34 \\
15.8\end{array}$ & 58 & $\begin{array}{l}1.62 \\
1.61\end{array}$ & $\begin{array}{l}0.24 \\
1.60\end{array}$ & 0 & $\begin{array}{l}0.24 \\
1.60\end{array}$ & 0 & 0 & $\begin{array}{l}0.24 \\
1.60\end{array}$ \\
\hline & & $49-50$ & $543-32-4,30$ to $543-34-2,10$ & 15.8 & 58 & 1.62 & 1.62 & 0 & $\begin{array}{l}1.60 \\
1.62\end{array}$ & 0 & 0 & 1.62 \\
\hline & & 50-51 & $543-34-2,10$ to $543 \mathrm{~A}-2-6,40$ & 15.8 & 57 & 1.66 & 1.70 & 0 & 1.70 & 0 & 0 & 1.70 \\
\hline & $\bar{z}$ & $51-52$ & $543 \mathrm{~A}-2-6,40$ to $543 \mathrm{~A}-4-4,20$ & 15.8 & s5 & 1.72 & 1.83 & 5 & 1.82 & 0 & 0 & 1.82 \\
\hline & & $52-53$ & $543 \mathrm{~A}-4-4,20$ to $543 \mathrm{~A}-6-1,150$ & 15.8 & 54 & 1.75 & 1.89 & 5 & 1.88 & 0 & 0 & 1.88 \\
\hline & Paleocene & $\begin{array}{l}53-54 \\
54-65\end{array}$ & $543 \mathrm{~A}-6-2,0$ to $543 \mathrm{~A}-6, \mathrm{CC}$ & 8.0 & 53 & 1.76 & 0.97 & 3 & 0.97 & 0 & 0 & 0.97 \\
\hline & & (65-66 -193 & $\begin{array}{l}\text { No Palecocene sediments corred } \\
543 \mathrm{~A}-7 \cdot 1,0 \text { to } 543 \mathrm{~A} \cdot-7.3,10\end{array}$ & $\begin{array}{l}0.0 \\
3.10\end{array}$ & 53 & 1.77 & $\begin{array}{l}0.0 \\
0.38\end{array}-1$ & $\overline{5}$ & 0.38 & 15 & 0.06 & 0.32 \\
\hline & & $66-67$ & $543 \mathrm{~A}-7.3,10$ to $543 \mathrm{~A}-7.5,20$ & 3.10 & 53 & 1.77 & 0.38 & 5 & 0.38 & is & 0.06 & 0.32 \\
\hline & & 67-68 & $543 \mathrm{~A}-7-5,20$ to $543 \mathrm{~A}-7, \mathrm{CC}$ & 3.10 & 52 & 1.78 & 0.39 & 5 & 0.38 & is & 0.06 & 0.32 \\
\hline & & $68-69$ & $543 \mathrm{~A}-7, \mathrm{CC}$ to $543 \mathrm{~A}-8-2,130$ & 3.10 & 52 & 1.78 & 0.39 & 5 & 0.38 & is & 0.06 & 0.32 \\
\hline & Cretaceous & $69-70$ & $543 \mathrm{~A}-8-2,130$ to $543 \mathrm{~A}-8.4,140$ & 3.10 & 52 & 1.78 & 0.39 & 5 & 0.38 & is & 0.06 & 0.32 \\
\hline & Cretactous & $70-71$ & $543 \mathrm{~A}-8-4,140$ to $543 \mathrm{~A}-8-6,6,150$ & 3.10 & s! & 1.79 & 0.39 & 5 & 0.39 & 15 & 0.06 & 0.33 \\
\hline & & $71-72$ & $543 \mathrm{~A}-8-7,0$ to $543 \mathrm{~A}-9-2,110$ & 3.10 & si & 1.79 & 0.39 & 5 & 0.39 & is & 0.06 & 0.33 \\
\hline & & $\begin{array}{l}72-73 \\
73-74\end{array}$ & 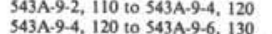 & $\begin{array}{l}3.10 \\
3.10\end{array}$ & $\begin{array}{c}51 \\
50\end{array}$ & $\begin{array}{l}1.79 \\
1.80\end{array}$ & $\begin{array}{l}0.39 \\
0.40\end{array}$ & 5 & $\begin{array}{l}0.39 \\
0.40\end{array}$ & ${ }_{15}^{15}$ & $\begin{array}{l}0.06 \\
0.06\end{array}$ & $\begin{array}{l}0.33 \\
0.34\end{array}$ \\
\hline & & 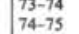 & 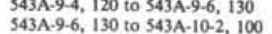 & $\begin{array}{l}3.10 \\
3.10\end{array}$ & $\begin{array}{l}50 \\
50\end{array}$ & $\begin{array}{l}1.80 \\
1.80\end{array}$ & $\begin{array}{l}0.40 \\
0.40\end{array}$ & $\begin{array}{c}5 \\
5\end{array}$ & $\begin{array}{l}0.40 \\
0.40\end{array}$ & 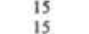 & $\begin{array}{l}0.06 \\
0.06\end{array}$ & $\begin{array}{l}0.34 \\
0.34\end{array}$ \\
\hline
\end{tabular}

Note: - indicates no data available. 
nearby Site 541, I estimate that Quaternary dip-corrected bulk sediment accumulation rates range from $4.6-5.3 \mathrm{~g} /\left(\mathrm{cm}^{2} \cdot 10^{3} \mathrm{yr}\right.$.) (Table 3$)$.

Pliocene (1.8-5 Ma) marly calcareous oozes, drilled from 173.5 to $226 \mathrm{~m}$ sub-bottom, have dip-corrected bulk sediment accumulation rates ranging from 1.7 to $5.6 \mathrm{~g} /$ $\left(\mathrm{cm}^{2} \cdot 10^{3}\right.$ yr.). The Pliocene 3- to 4-Ma sediment accumulation rates were calculated by assuming that the biostratigraphic inversion between Cores 542A-1 and 542-1 is located at the midpoint of the uncored interval (183$202 \mathrm{~m}$ ) between them (Fig. 3). The 4- to 5-Ma sediment accumulation rates are more tightly controlled biostratigraphically and may be lower than the 3- to 4-Ma interval rates due to a smaller contribution of calcareous components documented by a decrease in carbonate content (Fig. 3; Table 1).

Late Miocene (5-7 Ma) marly calcareous oozes and muds were drilled from 226 to $325.5 \mathrm{~m}$ sub-bottom and have dip-corrected bulk sediment accumulation rates ranging from 1.3 to $6.8 \mathrm{~g} /\left(\mathrm{cm}^{2} \cdot 10^{3} \mathrm{yr}\right.$.) (Table 3). Cored intervals barren of microfossils decrease the accuracy of these Miocene sediment accumulation rate determinations. In addition, the documented biostratigraphic inversion between Cores 542A-8 and 542A-9 had to be accounted for in calculating sediment accumulation rates for the 6- to 7-Ma interval. This calculation required the assumption that the boundary between nannofossil Zones CN9b and CN9a (6.6 Ma) is located at the midpoint of the Core 542A-2 barren interval, that the barren interval in Cores 542A-4 and 542A-5 contains no biostratigraphic inversions, and that the bottom of biostratigraphic Zone CN9a (7 Ma) is located at the base of Core $542 \mathrm{~A}-7$ above the biostratigraphic inversion between Cores 542A-8 and 542A-9.

Carbonate percentages range from 5 to $30 \%$ in Site 542 sediments (Tables 1 and 2). These percentages produce calculated carbonate accumulation rates of 0.2 to $1.7 \mathrm{~g} /\left(\mathrm{cm}^{2} \cdot 10^{3} \mathrm{yr}\right.$ ) (Fig. 3; Table 3).

\section{Site 543}

Two holes were drilled at Site 543, the oceanic reference site located $3.5 \mathrm{~km}$ east of the accretionary prism toe on the northern edge of the Tiburon Rise. Hole 543 was cored continuously for $324 \mathrm{~m}$ (8-332 m sub-bottom) before the core barrel jammed and the hole had to be abandoned. One mudline core $10 \mathrm{~m}$ long was drilled from 0 to $10 \mathrm{~m}$ sub-bottom in Hole $543 \mathrm{~A}$, and continuous cores were taken below $332 \mathrm{~m}$ sub-bottom. Hole 543A penetrated Upper Cretaceous ( $75 \mathrm{Ma}$; Bergen, this volume) basaltic basement rocks at $411 \mathrm{~m}$ sub-bottom. The youngest sediment continuously cored at Hole 543A (Core 543A-2) is stratigraphically equivalent to the oldest sediment cored at Hole 543 (Core 543-34). Core recovery in Hole 543 ranged from 0 to $103 \%$ (values exceeding $100 \%$ represent forcing of material outside the core diameter up the core by the larger face of the core bit), and averaged $79.5 \%$; core recovery of the sediment drilled in Hole 543A (Core 1-Sample 543A-10-2, 100 $\mathrm{cm}$ ) ranged from 11 to $79 \%$, averaging only $34 \%$.

Intervals barren of calcareous and siliceous microfossils are found at 77 to $161 \mathrm{~m}$ (Cores 543-11 to 543-16) and 370 to $379.5 \mathrm{~m}$ (Core 543A-6) sub-bottom. The interval from 313 to $370 \mathrm{~m}$ sub-bottom (Cores 543-33 to $543 \mathrm{~A}-5$ ) contains only very poorly preserved radiolarians, which, at the time of this writing, could not be dated more accurately than "early Eocene" (Renz, this volume). Barren intervals and cores with poor or no core recovery (e.g., Cores 543-21, 543-22, 543A-6, 543A-8, and 543A-9) provide little biostratigraphic control, and 1-Ma intervals interpolated from these cores must be considered rough estimations only. No obvious faulting, folding, or repetition of biostratigraphic zonations was observed at Site 543 , and bedding attitudes are everywhere less than or equal to $5^{\circ}$. Calculated sediment accumulation rates may therefore closely approximate actual rates of sediment deposition (Fig. 4; Table 3).

Thirty-seven meters of Quaternary $(0-1.8 \mathrm{Ma})$ muds and nannofossil muds were drilled in Core 543A-1 and Cores 543-1 through 543-3. Assuming that the surface sediment cored in 543A-1 is modern sediment, 1-Ma-interval Quaternary dip-corrected bulk sediment accumulation rates range from 1.7 to $2.4 \mathrm{~g} /\left(\mathrm{cm}^{2} \cdot 10^{3} \mathrm{yr}\right.$. $)$.

Pliocene (1.8-5 Ma) muds and nannofossil muds were drilled from 37.5 to $87 \mathrm{~m}$ sub-bottom and have dip-corrected bulk sediment accumulation rates ranging at 1 Ma intervals from 0.8 to $2.1 \mathrm{~g} /\left(\mathrm{cm}^{2} \cdot 10^{3} \mathrm{yr}\right.$.). Biostratigraphic control of the 2- to 3-Ma-interval bulk accumulation rate of $2.1 \mathrm{~g} /\left(\mathrm{cm}^{2} \cdot 10^{3} \mathrm{yr}\right.$.) is good, whereas the lower accumulation rate of $0.8 \mathrm{~g} /\left(\mathrm{cm}^{2} \cdot 10^{3} \mathrm{yr}\right.$.) is based on poorly defined biostratigraphic zonations and is likely too low.

Site 543 Miocene (5-23 Ma) muds and radiolarian clays were drilled from 87 to $218 \mathrm{~m}$ sub-bottom (Sample 5439-2, $45 \mathrm{~cm}$ to Section 543-22,CC), and have low dip-corrected bulk sediment accumulation rates ranging from 0.4 to $0.7 \mathrm{~g} /\left(\mathrm{cm}^{2} \cdot 10^{3} \mathrm{yr}\right.$.). Upper Miocene sediments are barren of microfossils, and core recovery in lowest Miocene sediments was poor. Therefore sediment accumulation rates calculated for these intervals have poor biostratigraphic control.

Oligocene (23-38 Ma) radiolarian clays and manganese-stained radiolarian clays drilled from 218 to $260 \mathrm{~m}$ sub-bottom (Core 543-23 to Sample 543-27-5, $140 \mathrm{~cm}$ ) have very low dip-corrected bulk sediment accumulation rates that range at 1-Ma intervals from 0.2 to $1.2 \mathrm{~g} /\left(\mathrm{cm}^{2}\right.$ - $10^{3} \mathrm{yr}$.), but average only $0.3 \mathrm{~g} /\left(\mathrm{cm}^{2} \cdot 10^{3} \mathrm{yr}\right.$.). As the radiolarian biostratigraphic zones used to determine Oligocene age-versus-depth relationships have an average duration of about $6 \mathrm{Ma}$, sediment accumulation rates calculated at 1-Ma intervals are the product of extensive interpolation that assumes constant sedimentation rates within the entire time interval represented by each biostratigraphic zone.

Eocene (38-53.5 Ma) sediments were drilled from 260 to $379 \mathrm{~m}$ sub-bottom and, except for the early Eocene, have low sediment accumulation rates. Late Eocene manganese-stained radiolarian clays have very low dip-corrected bulk sediment accumulation rates of $0.2 \mathrm{~g} /\left(\mathrm{cm}^{2}\right.$. $10^{3}$ yr.). Middle Eocene manganese-stained radiolarian clays also have low dip-corrected bulk sediment accumulation rates ranging from 0.1 to $1.6 \mathrm{~g} /\left(\mathrm{cm}^{2} \cdot 10^{3} \mathrm{yr}\right.$.). The radiolarian Zone $P$. Goetheana is missing from 
middle Eocene sediments, indicating either a period of nondeposition or erosion (Renz, this volume). Early Eocene claystones and zeolitic claystones have dip-corrected bulk sediment accumulation rates ranging from 1.0 to $1.9 \mathrm{~g} /\left(\mathrm{cm}^{2} \cdot 10^{3} \mathrm{yr}\right.$.), but rates estimated from this interval have poor biostratigraphic control.

Within the biostratigraphic resolution possible in Site 543 cores, no Paleocene (53.5-65 Ma) sediments were drilled in either Hole 543 or 543A. Early Eocene claystones directly overlie Cretaceous calcareous ferruginous claystones, indicating a Paleocene period of nondeposition, or very slow deposition below the $\mathrm{CCD}$, or an erosional hiatus. Pinch-outs commonly observed in Site 543 seismic data support the presence of hiatuses in this portion of the stratigraphic section (see Site 543 report, this volume).

Cretaceous calcareous ferruginous claystones were drilled from 379 to $411 \mathrm{~m}$ sub-bottom, nonconformably overlying Cretaceous basaltic basement rocks. These Cretaceous sediments have an estimated dip-corrected bulk sediment accumulation rate of $0.4 \mathrm{~g} /\left(\mathrm{cm}^{2} \cdot 10^{3}\right.$ yr.). This rate is based on an estimated basement age of $75 \mathrm{Ma}$ and assumes that sediment deposition began soon after the basalt pillows formed and was continuous and is preserved up to the Cretaceous/Tertiary boundary. The biostratigraphic age of these Cretaceous sediments based on nannofossil zonations (Verbeck, 1976) is very poorly controlled due to poor core recovery and poor preservation of incomplete nannofossil assemblages (Bergen, this volume). Assumptions made in determining sediment accumulation rates for the Cretaceous intervals may therefore be wrong, and the true sediment accumulation rates may be significantly different (probably higher, as might be expected due to deposition above the CCD) than those reported here.

The only Site 543 sediments that contain carbonate components are the Pleistocene and Pliocene ashy nannofossil muds (Core 543-1 to Section 543-7-2) and the Cretaceous calcareous ferruginous claystones (Core 543A7 to Sample 543A-10-2, $100 \mathrm{~cm}$ ). The carbonate content of the Pleistocene and Pliocene ashy nannofossil muds ranges from 5 to $15 \%$, corresponding to carbonate ac- cumulation rates of approximately 0.1 to $0.4 \mathrm{~g} /\left(\mathrm{cm}^{2}\right.$. $10^{3}$ yr.) (Tables 1 and 2; Fig. 4). The carbonate content of the Cretaceous calcareous ferruginous claystones averages $15 \%$, producing carbonate accumulation rates of about $0.1 \mathrm{~g} /\left(\mathrm{cm}^{2} \cdot 10^{3} \mathrm{yr}\right.$.). Early Pliocene through Eocene sediments do not contain any significant carbonate component.

\section{DISCUSSION}

Crude sedimentation rates (in $\mathrm{m} / \mathrm{Ma}$ ) determined from biostratigraphic age-versus-depth information were calculated on board ship for each Leg 78A site and are published in Moore, Biju-Duval, et al. (1982) and BijuDuval, Moore, et al. (1981). Though these sedimentation rates are gross estimates at best and do not account for downhole porosity or density changes or tectonic tilting, they are surprisingly similar to the dip-corrected bulk sediment accumulation rates presented in this chapter. Table 4 is a summary comparison of the sedimentation rates determined on board ship and the more carefully determined sedimentation rates and sediment accumulation rates presented in this study. Obviously, shipboard calculation of sedimentation rates provides useful information and should continue to be routinely calculated by DSDP scientists, especially at drill sites that have undergone little tectonic tilting (e.g., oceanic reference sites), and where downhole porosity and density gradients are not too great.

The sediments drilled at Sites 541 and 542 are lithologically and paleontologically similar to the Neogene section drilled at Site 543 . This similarity supports the interpretation of offscraping and incorporation of the Neogene sedimentary section into the Lesser Antilles accretionary prism. Even after making corrections for all small-scale faults that could be documented by biostratigraphic repetitions, the dip-corrected bulk sediment accumulation rates calculated for lower-slope Sites 541 and 542 are significantly greater than the rates calculated for the corresponding Neogene section in oceanic reference Site 543 (Fig. 5). This difference is especially true of the upper Miocene section cored at each site. The higher rates observed at Sites 541 and 542 could

Table 4. Comparison of various Leg $78 \mathrm{~A}$ sedimentation rate calculations.

\begin{tabular}{|c|c|c|c|c|c|c|c|c|c|c|c|c|}
\hline \multirow[b]{2}{*}{ Age } & \multicolumn{4}{|c|}{$\begin{array}{l}\text { Sedimentation rates } \\
\text { calculated on board ship } \\
(\mathrm{m} / \mathrm{Ma})^{\mathrm{b}}\end{array}$} & \multicolumn{4}{|c|}{$\begin{array}{c}\text { Sedimentation rates } \\
(\mathrm{m} / \mathrm{Ma}) \\
\text { (this study) }\end{array}$} & \multicolumn{4}{|c|}{$\begin{array}{l}\text { Dip-corrected bulk } \\
\text { sediment accumulation rates } \\
{\left[\mathrm{g} /\left(\mathrm{cm}^{2} \cdot 10^{3} \mathrm{yr}\right)\right]}\end{array}$} \\
\hline & $\begin{array}{l}\text { Site } \\
541 \\
(\mathrm{~A})^{\mathrm{a}}\end{array}$ & $\begin{array}{l}\text { Site } \\
541 \\
(\mathrm{~B})^{\mathrm{a}}\end{array}$ & $\begin{array}{l}\text { Site } \\
\text { S42 }\end{array}$ & $\begin{array}{l}\text { Site } \\
543\end{array}$ & $\begin{array}{l}\text { Site } \\
541 \\
(\mathrm{~A})^{\mathrm{a}}\end{array}$ & $\begin{array}{l}\text { Site } \\
541 \\
(B)^{\mathrm{a}}\end{array}$ & $\begin{array}{l}\text { Site } \\
541\end{array}$ & $\begin{array}{l}\text { Site } \\
541\end{array}$ & $\begin{array}{l}\text { Site } \\
541 \\
(A)^{\mathrm{a}}\end{array}$ & $\begin{array}{l}\text { Site } \\
541 \\
(B)^{\mathrm{a}}\end{array}$ & $\begin{array}{l}\text { Site } \\
542\end{array}$ & $\begin{array}{l}\text { Site } \\
543\end{array}$ \\
\hline Quaternary & 40 & & $50-60^{\mathrm{C}}$ & 20 & $50-55$ & & $50^{c}$ & $20-26$ & $4.9-6.1$ & & $4.6-5.3^{c}$ & $1.7-2.4$ \\
\hline Pliocene & $<43^{c}$ & & $<46^{\mathrm{c}}$ & $13-15$ & $16-35^{\mathrm{c}}$ & $14-23^{c}$ & $14-50^{c}$ & $9-22$ & $2.5-3.8^{\mathrm{c}}$ & $1.8-2.8^{\mathrm{C}}$ & $1.7-5.6^{c}$ & $0.8-2.1$ \\
\hline $\begin{array}{l}\text { late Miocene } \\
\text { middle Miocene }\end{array}$ & $<12-13^{c}$ & $\begin{array}{r}13^{c} \\
0^{d}\end{array}$ & $<14^{c}$ & $\underset{6-7}{14-15}$ & $22-38^{\mathrm{C}}$ & $\frac{7-41^{c}}{7}$ & $11-61^{c}$ & 9 & $3.6-4.2^{\mathrm{c}}$ & $\begin{array}{l}0.6-4.4^{c} \\
0.6\end{array}$ & $1.3-6.8^{\mathrm{c}}$ & $\begin{array}{l}0.7 \\
0.6\end{array}$ \\
\hline early Miocene & & & & 7 & & & & 4-8 & & & & $0.4-0.6$ \\
\hline Oligocene & & & & 2.5 & & & & $2-11$ & & & & $0.2-1.2$ \\
\hline late Eocene & & & & 7 & & & & 2 & & & & 0.2 \\
\hline middle Eocene & & & & 1.4 & & & & $1-2$ & & & & $0.1-0.8$ \\
\hline carly Eocene & & & & 17 & & & & 15 & & & & $1.6-1.9$ \\
\hline Paleocene & & & & $0^{\mathrm{e}}$ & & & & $0^{c}$ & & & & $0^{e}$ \\
\hline Late Cretaceous & & & & $>2.3$ & & & & 3 & & & & 0.4 \\
\hline
\end{tabular}

Note: Blank spaces indicate no data available.

a Distinction of the two tectonic units (Tectonic Units A and B) drilled at Site 541

b Published in Moore, Biju-Duval, et al. (1982) and Biju-Duval, Moore, et al. (1981).

c Probably higher because of small stratigraphic inversions, and faulted and folded beds.

d Probably missing or thinned in the décollement.

e Not recovered (hiatus?). 


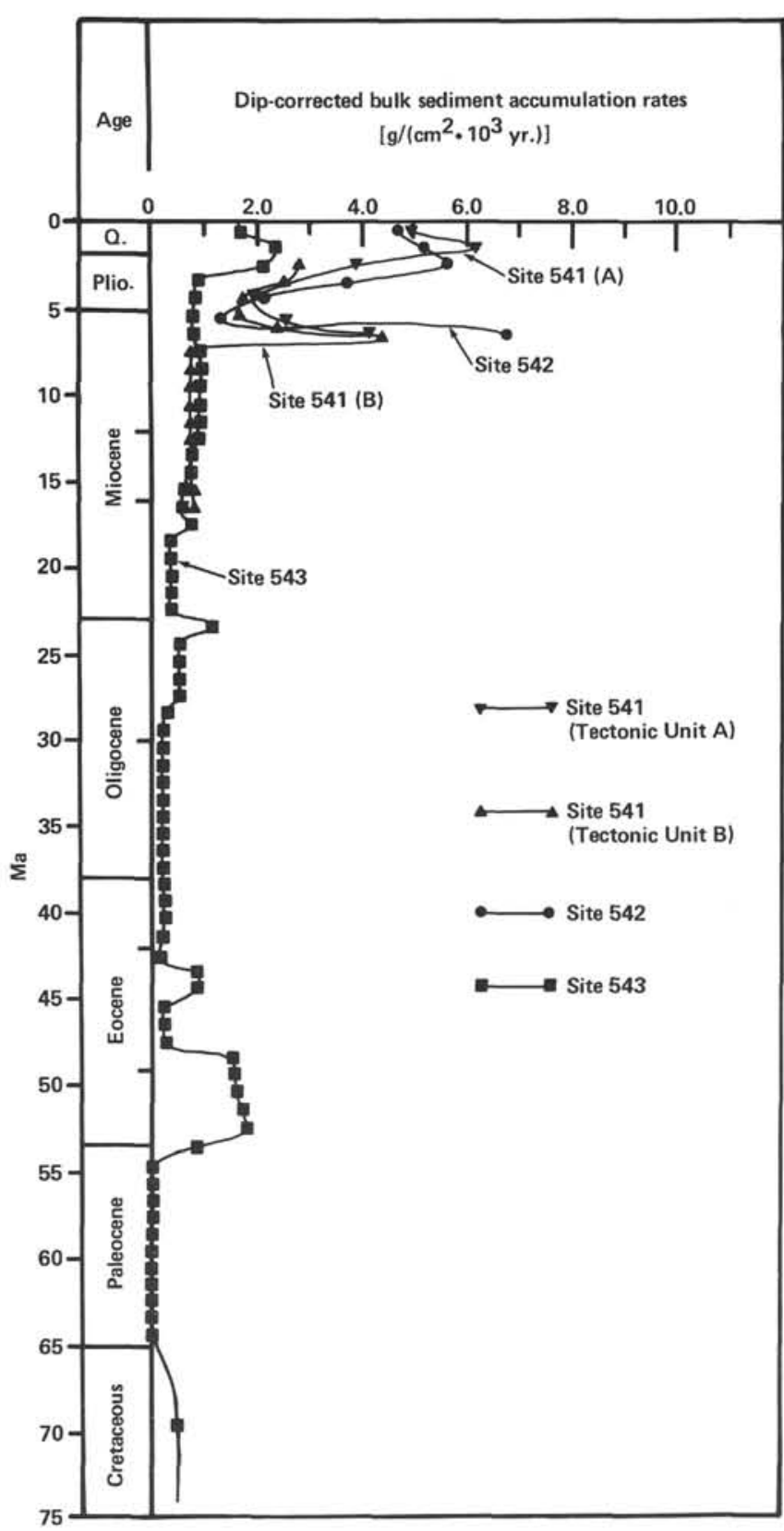

Figure 5. Dip-corrected bulk sediment accumulation rates for DSDP Leg 78A Sites 541, 542, and 543. (Neogene rates at Sites 541 and 542 are significantly greater than Neogene rates at Site 543.)

result from a number of causes: (1) tectonic thickening in the accretionary prism due to layer-parallel shortening and/or folding or faulting on too small a scale to be documented by any biostratigraphic inversions or repetitions (Cowan et al., this volume); (2) deposition in slightly shallower water farther above the CCD, resulting in preservation of a greater percentage of calcareous microfossils, an explanation supported by the greater percentage of carbonate found in the Neogene sediments of Sites 541 and 542 (Tables 1 and 2; Moore, Biju-Duval, et al., 1982); and (3) a greater percentage of foraminiferal gravity flows in Sites 541 and 542 due to closer proximity to the trench slope (Hemleben and
Troester, this volume). An estimate of the amount of tectonic thickening that could have taken place in Site 541 and 542 upper Miocene sediments can be made by dividing the average late Miocene sediment accumulation rate observed at these sites by that observed at Site 543. This calculation (which gives a maximum value for the amount of structural thickening that has occurred by assuming that the thickness of Sites 541 and 542 upper Miocene sediments before accretion was similar to that now observed at oceanic reference Site 543) indicates that the upper Miocene sediments at Sites 541 and 542 have been structurally thickened by the process of accretion to five times their original thickness.

The dip-corrected bulk sediment accumulation rates calculated at Site 543 range from 0.1 to $2.4 \mathrm{~g} /\left(\mathrm{cm}^{2} \cdot 10^{3}\right.$ yr.), which falls within the range typical for oceanic pelagic sediments (van Andel et al., 1975; Worsley and Davies, 1979a; Sigurdsson et al., 1980; Shephard and McMillen, 1982). Lack of terrigenous material at Site 543 could be due to one or both of two factors: (1) great distance from South American terrigenous sediment sources; and (2) isolation from South American terrigenous material due to location of Site 543 on the northern slope of the Tiburon Rise (Chase and Bunce, 1969; Peter and Westbrook, 1976; Moore, Biju-Duval, et al., 1982). I prefer the latter explanation, as Site 27 , located a comparable distance from South America, contains terrigenous turbidites (Bader et al., 1970a). However, piston cores taken in the area near Site 543 but off of the Tiburon Rise consist dominantly of pelagic and hemipelagic muds, indicating a general lack of terrigenous turbidite deposition from South American sediment sources at the latitude of Site 543 at least during the Quaternary (see Wright, this volume).

Additional sediment accumulation rate information for the Lesser Antilles intraoceanic island arc region is available from other sources, as discussed later. The piston cores provide information about Quaternary sediment accumulation rates. Sigurdsson et al. (1980) report sediment accumulation rates of 10.8 to $15.8 \mathrm{~g} /\left(\mathrm{cm}^{2}\right.$. $10^{3}$ yr.) $\left(13-19 \mathrm{~cm} / 10^{3} \mathrm{yr}\right.$.) for the last $10,000 \mathrm{yr}$. in sediments cored west of the island arc in Grenada Basin, and 0.6 to $4.2 \mathrm{~g} /\left(\mathrm{cm}^{2} \cdot 10^{3} \mathrm{yr}\right.$.) for the same time period in sediment cored east of the island arc on the accretionary prism and Atlantic abyssal plain. Damuth (1977) compares Holocene interglacial (0-11,000 yr. ago) and Wisconsin glacial $(11,000-85,000$ yr. ago) accumulation rates in western equatorial Atlantic piston cores. He reports the following Holocene accumulation rates: 3.33 to $5.83 \mathrm{~g} /\left(\mathrm{cm}^{2} \cdot 10^{3} \mathrm{yr}\right.$. $)\left(4-7 \mathrm{~cm} / 10^{3} \mathrm{yr}\right.$. $)$ for the middle and lower continental slope; 1.67 to $3.33 \mathrm{~g} /\left(\mathrm{cm}^{2} \cdot 10^{3}\right.$ yr.) $\left(2-4 \mathrm{~cm} / 10^{3}\right.$ yr.) for abyssal regions above the CCD; and, 0.83 to $1.67 \mathrm{~g} /\left(\mathrm{cm}^{2} \cdot 10^{3} \mathrm{yr}\right.$.) $\left(1-2 \mathrm{~cm} / 10^{3} \mathrm{yr}\right.$.) for abyssal regions below the $\mathrm{CCD}$. He estimates that the Wisconsin glacial sedimentation rate in the Lesser Antilles forearc region is $4.16 \mathrm{~g} /\left(\mathrm{cm}^{2} \cdot 10^{3} \mathrm{yr}\right.$. $)\left(5 \mathrm{~cm} / 10^{3} \mathrm{yr}\right.$. $)$. The Quaternary accumulation rates reported by Sigurdsson et al. (1980) and Damuth (1977) compare well with the Quaternary accumulation rates calculated for Leg $78 \mathrm{~A}$ sediments of the same age. 
Three DSDP sites, other than those drilled on Leg $78 \mathrm{~A}$, have been drilled in the Lesser Antilles intraoceanic island arc region (Fig. 1): (1) Site 27 (Leg 4), drilled approximately $400 \mathrm{~km}$ east of the arc in the western Atlantic Basin immediately south of the Barracuda Rise (Bader et al., 1970a); (2) Site 30 (Leg 4), drilled on the Aves Ridge west of the arc (Bader et al., 1970b); and (3) Site 148 (Leg 15), drilled on the western edge of the Aves Ridge approximately $50 \mathrm{~km}$ northwest of Site 30 (Edgar, Saunders, et al., 1973). Sediment accumulation rates for Site 27 pelagic sediments average $1.19 \mathrm{~g} /\left(\mathrm{cm}^{2} \cdot 10^{3} \mathrm{yr}\right.$. $)$ $\left(1.15 \mathrm{~cm} / 10^{3} \mathrm{yr}\right.$.), which is a bit higher than that typical of similar sediments drilled at Site 543. Rates calculated for Site $\mathbf{2 7}$ are, however, only rough estimates, because the site was not continuously cored. Bulk sediment accumulation rates for Site 30 systematically decrease downcore (Benson, Gerard, and Hay, 1970). Pleistocene volcanic silts were deposited at rates averaging $11.34 \mathrm{~g} /$ $\left(\mathrm{cm}^{2} \cdot 10^{3}\right.$ yr.) $\left(10 \mathrm{~cm} / 10^{33} \mathrm{yr}\right.$.); Pliocene volcanic silts and hemipelagics were deposited at rates averaging 5.14 $\mathrm{g} /\left(\mathrm{cm}^{2} \cdot 10^{3} \mathrm{yr}\right.$.) $\left(4.0 \mathrm{~cm} / 10^{3} \mathrm{yr}\right.$. $)$; and Miocene pelagic and hemipelagic sediments were deposited at rates averaging $0.51 \mathrm{~g} /\left(\mathrm{cm}^{2} \cdot 10^{3} \mathrm{yr}\right.$.) $\left(0.4 \mathrm{~cm} / 10^{3} \mathrm{yr}\right)$. The increasing sediment accumulation rates upcore reflect increasing volcanic input from the Lesser Antilles island arc with time. The Site 30 Pleistocene sedimentation rates compare well with the rates calculated for Quaternary sediments west of the island arc by Sigurdsson et al. (1980). Site 148 , located close to Site 30, contains 249 $\mathrm{m}$ of lower Pliocene-upper Pleistocene pelagic calcareous clays and marls unconformably overlying $23 \mathrm{~m}$ of reworked, indurated volcanic sands (Paleocene?). Sediment accumulation rates for Site 148 sediments range from 4.15 to $6.52 \mathrm{~g} /\left(\mathrm{cm}^{2} \cdot 10^{3} \mathrm{yr}\right.$.) $\left(3.15-5.5 \mathrm{~cm} / 10^{3}\right.$ yr.), comparable with the rates calculated for Site $30 \mathrm{vol}$ canic silts and hemipelagics. DSDP drilling documents the greater sediment accumulation rates typical of regions west, rather than east, of the arc due to greater influx of pyroclastic debris flows from the Lesser Antilles island arc in that direction (Sigurdsson et al., 1980; see Wright, this volume).

Detailed sediment accumulation rate calculations have not been routinely made for DSDP active margin sites, with the exception of Leg 66 sites in the Middle American convergent margin off central Mexico (Shephard and McMillen, 1982). The maximum accumulation rates of the Pliocene and Quaternary deposits in lower slope Sites 541 and 542 are at the lower end of the range of values observed at other convergent margin lower slope settings (von Huene and Kulm, 1973; Arthur et al., 1980; von Huene, Aubouin, et al., 1980; Moore, Watkins, et al., 1981; Shephard and McMillen, 1982). This observation reflects the intraoceanic setting of the Lesser Antilles arc, where little terrigenous material is contributed to the Leg 78A area. Published sedimentation models for active continental convergent margins (Schweller and Kulm, 1978; Underwood et al., 1980) all indicate that large amounts of terrigenous materials are deposited in trench and trench slope basin settings via submarine canyons fed by erosion of the adjacent continental block. Such settings have correspondingly high sediment accumulation rates. These models are, however, apparently not valid for the northern Lesser Antilles or similar intraoceanic active margin settings distant from terrigenous sediment sources. New models must be derived to describe the sedimentation patterns and depositional processes operating in these settings.

\section{ACKNOWLEDGMENTS}

This manuscript was reviewed by Tom A. Davies, Robert E. Garrison, and Kenneth J. McMillen. Valuable comments were added by $\mathrm{Ca}$ sey Moore, Eli Silver, and Jill Whitman. Shipboard chemist David Ripley provided the "Karbonate Bombe" measurements, Blair McKay ran shore-based LECO samples, and William Meyer provided techni$\mathrm{cal}$ assistance with the calculation of sediment accumulation rates. Partial support for this study came from Joint Oceanographic Institutions, Inc. as part of a synthesis study of the sedimentology of the Lesser Antilles intraoceanic island arc.

\section{REFERENCES}

Arthur, M. A., von Huene, R., and Adelseck, C. G., 1980. Sedimentary evolution of the Japan fore-arc region off northern Honshu, Legs 56 and 57, Deep Sea Drilling Project. In Scientific Party, Init. Repts. DSDP, 56, 57, Pt. 1: Washington (U.S. Govt. Printing Office), 521-568.

Bader, R. G., 1970. Shore-based laboratory procedures. In Bader, R. G., Gerard, R. D., et al., Init. Repts. DSDP, 4: Washington (U.S. Govt. Printing Office), 745-753.

Bader, R. G., Gerard, R. D., et al., 1970a. Site 27. In Bader, R. G., Gerard, R. D., et al., Init. Repts. DSDP, 4: Washington (U.S. Govt. Printing Office), 93-123.

1970b. Site 30. In Bader, R. G., Gerard, R. D., et al., Init. Repts. DSDP, 4: Washington (U.S. Govt. Printing Office), 215-241.

Bennett, R. H., and Keller, G. H., 1973. Physical properties evaluation. In van Andel, Tj. H., Heath, G. R., et al., Init. Repts. DSDP, 16: Washington (U.S. Govt. Printing Office), 513-519.

Benson, W. E., Gerard, R. D., and Hay, W. W., 1970. Summary and conclusions. In Bader, R. G., Gerard, R. D., et al., Init. Repts. DSDP, 4: Washington (U.S. Govt. Printing Office), 659-673.

Biju-Duval, B., Moore, J. C., Blackinton, G., Bergen, J. A., Claypool, G. E., et al., 1981. Premiers résultats des forages IPOD implantés lors de la croisière 78A du Glomar Challenger au nord-est de la ride de la Barbade (arc des Petites Antilles) - tectonique frontale d'un prisme d'accrétion. C. R. Acad. Sci. Paris, 293:621-628.

Bode, G. W., and Cronan, D. S., 1973. Carbon and carbonate analyses, Leg 16. In van Andel, Tj. H., Heath, G. R., et al., Init. Repts. DSDP, 16: Washington (U.S. Govt. Printing Office), 521-528.

Boyce, R. E., 1976. Definitions and laboratory tecniques of compressional sound velocity parameters and wet-water content-wet-bulk density and porosity parameters by gravimetric and gamma-ray attenuation techniques. In Schlanger, S., Jackson, D., et al., Init. Repts. DSDP, 33: Washington (U.S. Govt. Printing Office), 931-957.

Boyce, R. E., and Bode, G. W., 1973. Carbon and carbonate analyses, Leg 9. In Hays, J. D., et al., Init. Repts. DSDP, 9: Washington (U.S. Govt. Printing Office), 797-816.

Bukry, D., and Okada, H., 1980. Supplementary modification and introduction of code numbers to the low-latitude coccolith biostratigraphic zonation. Mar. Micropaleontol., 5:321-325.

Chase, R. L., and Bunce, E. T., 1969. Underthrusting of the eastern margin of the Antilles by the floor of the western North Atlantic Ocean, and the origin of the Barbados Ridge. J. Geophys. Res., $74: 1413-1420$.

Damuth, J. E., 1977. Late Quaternary sedimentation in the western equatorial Atlantic. Geol. Soc. Am. Bull., 88:695-710.

Davies, T. A., Hay, W. W., Southarm, J. R., and Worsley, T. R., 1977. Estimates of Cenozoic oceanic sedimentation rates. Science, 197: 53-55.

Edgar, N. T., Saunders, J. B., et al., 1973. Init. Repts. DSDP, 15: Washington (U.S. Govt. Printing Office).

Evans, H. B. and Cotterell, C. H., 1970. Gamma ray attenuation density scanner. In Peterson, M. N. A., Edgar, N. T., et al., Init. Repts. DSDP, 2: Washington (U.S. Govt. Printing Office), 460-471.

Gartner, S., 1977. Calcareous nannofossil biostratigraphy and revised zonation of the Pleistocene. Mar. Micropaleontol., 2:1-25. 
Moore, J. C., Biju-Duval, B., Bergen, J. A., Blackinton, G., Claypool, G. E., et al., 1982. Offscraping and underthrusting of sediment at the deformation front of the Barbados Ridge-results from DSDP Leg 78A. Geol. Soc. Am. Bull., 93:1065-1077.

Moore, J. C., Watkins, J. S., et al., 1981. Facies belts of the Middle America Trench and forearc region, southern Mexico-results from Leg 66 DSDP. In Leggett, J. K. (Ed.), Trench and Forearc Sedimentation and Tectonics. Geol. Soc. London Spec. Publ., 10: 77-94.

Müller, G., and Gastner, M., 1974. The "Karbonate Bombe." Neues Jahrb. Mineral., H10:466-469.

Peter, G., and Westbrook, G. K., 1976. Tectonics of the southwestern North Atlantic and Barbados Ridge complex. Am. Assoc. Pet. Geol. Bull., 60:1078-1106.

Riedel, W. R., and Sanfilippo, A., 1978. Stratigraphy and evolution of tropical Cenozoic radiolarians. Micropaleontology, 23:61-69.

Sanfilippo, A., and Riedel, W. R., 1982. Revision of the radiolarian genera Theocotyle, Theocotylissa, and Thyrsocyrtic. Micropaleontology, 28(2): 170-188.

Schweller, W. J., and Kulm, L. D., 1978. Depositional patterns and channelized sedimentation in active eastern Pacific trenches. In Stanley, D. J., and Kelling, G. (Eds.), Sedimentation in Submarine Canyons, Fans, and Trenches: Stroudsburg, Pa. (Dowden, Hutchinson, and Ross), pp. 311-324.

Shephard, L. E., and McMillen, K. J., 1982. Sedimentation rates of the southern Mexico continental margin, DSDP Leg 66. In Moore, J. C., Watkins, J. S., et al., Init. Repts. DSDP, 66: Washington (U.S. Govt. Printing Office), 445-451.

Sigurdsson, H., Sparks, R. J. S., Carey, S., and Huang, T. C., 1980. Volcanogenic sedimentation in the Lesser Antilles arc. J. Geol., $88: 523-540$.
Underwood, M. B., Bachman, S. B., and Schweller, W. J., 1980. Sedimentary processes and facies associations within trench and trenchslope settings. In Field, M. E., et al. (Eds.), Quaternary Depositional Environments on the Pacific Continental Margin. Soc. Econ. Pal. Mineral., Pac. Sect., pp. 211-229.

van Andel, Tj. H., Heath, G. R., and Moore, T. C., 1975. Cenozoic history and paleoceanography of the central equatorial Pacific Ocean. Geol. Soc. Am. Mem. 143.

Verbeck, J. W., 1976. Calcareous nannoplankton biostratigraphy of middle and upper Cretaceous deposits in Tunisia, southern Spain, and France. Utrecht Micro. Bull., 16:1-157.

von Huene, R., Aubouin, J., et al., 1980. Leg 67, DSDP, Mid-America transect off Guatemala. Geol. Soc. Am. Bull., 91:421-432.

von Huene, R., and Kulm, L. D., 1973. Tectonic summary of Leg 18. In Kulm, L. D., von Huene, R., et al., Init. Repts. DSDP, 18: Washington (U.S. Govt. Printing Office), 961-976.

Whitman, J. M. and Davies, T. A., 1979. Cenozoic oceanic sedimentation rates-how good are the data? Mar. Geol., 30:269-284.

Worsley, T. R., and Davies, T. A., 1979a. Cenozoic sedimentation in the Pacific Ocean-steps toward a quantitative evolution. J. Sediment. Petrol., 49:1131-1148.

$1979 \mathrm{~b}$. Sea-level fluctuations and deep-sea sedimentation rates. Science, 203:455-456.

Date of Initial Receipt: August 3, 1982

Date of Acceptance: October 26, 1982 